BBA 46 I 88

\title{
THE IRON ELECTRON-NUCLEAR DOUBLE RESONANCE (ENDOR) OF TWO-IRON FERREDOXINS FROM SPINACH, PARSLEY, PIG ADRENAL. CORTEX AND PSEUDOMONAS PUTIDA*
}

\author{
J. FRITZ, R. ANDERSON, J. FEE**, G. PALMER AND R. H. SANDS
}

Biophysics Research Division, Institute of Science and Technology, University of Michigan, Ann Arbor, Mich. ${ }^{8} 8_{105}$ (U.S.A.)

J. C. M. TSIBRIS AND I. C. GUNSALUS

Department of Biochemistry, University of Illinois, Urbana, Ill. 6I8oI (U.S.A.)

W. H. ORME-JOHNSON AND H. BEINERT

Department of Biochemistry and the Institute for Ensyme Research, University of Wisconsin, Madison, Wisc. 53706 (U.S.A.)

(Received May 2 Ist, I97 I)

\section{SUMMARY}

The iron electron-nuclear double resonance (ENDOR) spectra of reduced iron-sulfur proteins (two-iron ferredoxins) from spinach, parsley, pig adrenal cortex and Pseudomonas putida unequivocally show two inequivalent iron atoms at the active sites of each of these proteins. The frequencies of the ENDOR lines establish the total electronic spin in the ground state to be $S=1 / 2$. The principal values of the hyperfine tensor have been determined for each of the iron atoms and these values are consistent with and lend considerable support to the model of a high-spin Fe(III) atom and a high-spin $\mathrm{Fe}$ (II) atom antiferromagnetically coupled to form an $S=1 / 2$ system. The measured principal axis components of the effective hyperfine tensors for $S=1 / 2$ are as follows ( $\mathrm{I}$ and 2 refer to the inequivalent iron sites):

Abbreviation: ENDOR, electron-nuclear double resonance.

* This is one of a series of papers describing the electronic properties of several of the twoiron-two-(labile)sulfur proteins; related publications contain the results of experiments on the Mössbauer ${ }^{25}$ (called II), magnetic susceptibility ${ }^{26}$ (called III) and infrared, optical and circular dichroism spectra ${ }^{27}$ (called IV). An integrated interpretation of all of the data in terms of a specific model for these proteins is being published separately. The substance of this paper (called I) on the electron-nuclear double resonance (ENDOR) spectrometry and that of II on the Mössbauer spectra of these proteins was presented at the 4 th International Conference on Magnetic Resonance in Biological Systems, Oxford, August, I970 (R. H. SANDS, J. Fritz, A. BEARDEN ANI W. R. I) UNHAM).

** I'resent address: Department of Chemistry, Rensselaer Polytechnic Institute, Troy, N.Y. I2180, U.S.A. 


\begin{tabular}{|c|c|c|c|c|c|c|c|}
\hline & \multicolumn{3}{|c|}{ Site $I(F e(I I I))$} & \multicolumn{4}{|c|}{ Site $2\left(F_{i}(I I)\right)$} \\
\hline & $A_{x}$ & $A_{y^{\prime}}$ & $A_{z}^{\prime}$ & $A_{x}$ & $A_{y}$ & $A_{z}$ & \\
\hline Spinach & $5 \mathrm{I} \pm \mathrm{I}$ & $50 \begin{array}{l}+2 \\
-7\end{array}$ & $42 \pm .1 .5$ & $?$ & $?$ & $35 \cdot 5 \stackrel{2}{ \pm}$ & $\mathrm{MHz}$ \\
\hline \multirow[t]{2}{*}{ Parsley } & $5^{I} \pm I$ & 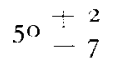 & $4^{2} \pm 2$ & $?$ & $?$ & $34.5 \pm 2.5$ & $\mathrm{MHz}$ \\
\hline & $A_{\perp}$ & $A_{\perp}^{\prime}$ & $A_{2}$ & $A_{\perp}$ & $A_{\perp}^{\prime}$ & $A_{z}$ & \\
\hline Adrenodoxin & $5^{0} \pm 1$ & $5^{6}+1$ & $43-1$ & I $7 \pm 4$ & $24 \mp 4$ & $35 \pm \mathrm{I} .5$ & $\mathrm{MHz}$ \\
\hline Putidlaredoxin & $5^{0} \pm 1.5$ & $\begin{array}{r}56+I \\
-3\end{array}$ & 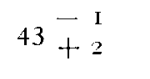 & $17 \pm 4$ & $24 \mp 4$ & $35 \pm 1.5$ & $\mathrm{MHz}$ \\
\hline
\end{tabular}

These data are consistent with Site I being ferric and Site 2, ferrous iron. The primes indicate that the $A$-tensor principal axes for Site I (Fe(III)) are apparently rotated about the $x$-axis with respect to the $g$-tensor axes by an angle $\theta\left(20^{\circ} \leqslant \theta \leqslant 40^{\circ}\right)$. The orientations of the $A$-tensors for Site 2 (Fe(II)) have not been determined and hence the values presented are the observed values of the $A$-tensors along the $x, y$, and $z$-axes of the $g$-tensor for this complex.

A brief introduction to the theory of ENDOR is given.

\section{INTRODUCTION}

Two-iron two-(labile)sulfur proteins or two-iron "ferredoxins", low molecular weight proteins of unusually low reduction potential, have been found to serve as electron carriers in a variety of biochemical reactions. The "two-iron" or "plant" type protein was first isolated from spinach chloroplasts, but is now known to be present in all examined photosynthetic organisms which utilize water as an electron donor and produce oxygen ${ }^{1}$. The "two-iron" proteins are found in plants, bacteria and mammals ${ }^{2,3}$. No three-dimensional structure information is available for these twoiron ferredoxins although the amino acid sequence has been published for a number of these proteins - these data show that the number of amino acid residues varies between ' 97 and II2 depending upon the species ${ }^{4-7}$.

Magnetic susceptibility measurements on the oxidized forms of several of the two-iron ferredoxins show that these proteins are diamagnetic at low temperatures. EPR and magnetic susceptibility measurements on the reduced forms show an effective $S=1 / 2$ molecular paramagnetism at temperatures below ${ }_{100}{ }^{\circ} \mathrm{K}^{8-11}$. Careful titration of the number of reducing equivalents show clearly that these proteins act as one electron-transfer agents; the quantitations of the EPR signal and the magnetic susceptibility measurements below $77^{\circ} \mathrm{K}$ are consistent with a single unpaired electron thus suggesting that the reducing electron is the paramagnetic electron; this suggestion will be examined in detail later. The EPR is observable only at temperatures below $200^{\circ} \mathrm{K}$ (lower still for spinach and parsley) and is not found for 
iron in typical environments. In all cases, two of the three $g$ values lie below 2.0023 (the $g$ value of the free electron) and the third $g$ value is only slightly above. Careful analysis of EPR spectra from ${ }^{57} \mathrm{Fe}$ and ${ }^{33} \mathrm{~S}$ enriched proteins ${ }^{12-18}$ have shown that the paramagnetic electron in the reduced form of these proteins interacts through the electron-nuclear hyperfine interaction with iron and with "acid-labile" and one or more cysteine or methionine sulfur atoms. The nature of the iron in the active sites of these proteins has been the subject of much speculation and several models have been proposed in the literature $\mathbf{1 9 - 2 4}^{\mathbf{2}}$. It is to this problem that this paper (called 1 ) on the electron-nuclear double resonance (ENDOR) spectrometry of these proteins is directed as well as the papers to follow on the Mössbauer spectrometry ${ }^{25}$ (II) magnetic susceptibility ${ }^{26}$ (III) and infrared, optical and circular dichroism spectra ${ }^{2 r}$ (IV). It is our belief that the results reported in these four papers clearly delineate the nature of the iron in these proteins and establish stringent criteria ${ }^{28}$ by which any future proposal for the structure of the active site of these proteins must be evaluated.

MATERIALS

Spinach ferredoxin was prepared as described by PETERING AND PALMER ${ }^{29}$, It was freed of iron and sulfide and then reconstituted with either ${ }^{57} \mathrm{Fe}$ or ${ }^{56} \mathrm{Fe}$ as follows: $4 \mathrm{ml}$, of approximately I mM ferredoxin were mixed with $1.0 \mathrm{ml} \mathrm{I} \mathrm{M} \mathrm{KOH}$. When the solution assumed a pale green color $64 \mathrm{mg}$ Tiron (catechol disulfonate) were added and the mixture incubated for $5 \mathrm{~min}$. The protein was acidified by the addition of $2 \mathrm{ml}$ of $20 \%$ trichloroacetic acid and the precipitate thus formed collected by low-speed centrifugation at room temperature. The precipitate was dissolved in a minimum volume of $0.7 \mathrm{M}$ Tris chloride, $\mathrm{pH} 7.3$, and reprecipitated 2 times with $5 \%$ trichloroacetic acid, and finally dissolved in $20 \mathrm{ml}$ of $0.2 \mathrm{M}$ Tris chloride, $\mathrm{pH} 7 \cdot 3$. To this solution were added in the following order, a 25 -fold molar excess of dithiothreitol, and a 2.5-fold molar excess of both $\mathrm{FeCl}_{2}$ and $\mathrm{Na}_{2} \mathrm{~S}$. The mixture was incubated aerobically at room temperature for $90 \mathrm{~min}$ with occasional gentle shaking. The reaction mixture was applied to a $\mathrm{I} \mathrm{cm} \times 2 \mathrm{~cm} \mathrm{DE}-52$ column which was then washed with $5^{0} \mathrm{ml}$ of $0 . \mathrm{I} 5 \mathrm{M}$ Tris chloride and the protein finally eluted with the same buffer containing I $\mathrm{M} \mathrm{NaCl}$. The brown eluate was passed over a $2 \mathrm{~cm} \times 30 \mathrm{~cm}$ column of Biogel P6o which was developed with 0.I5 M Tris chloride. The red-brown eluate was concentrated by absorption on a $\mathrm{I} \mathrm{cm} \times 2 \mathrm{~cm} \mathrm{DE}-52$ column and later eluted slowly with $0 .{ }_{5} \mathrm{M}$ Tris chloride containing I $\mathrm{M} \mathrm{NaCl}$. The sample was then applied to a $2 \mathrm{~cm} \times$ I $2 \mathrm{~cm}$ Sephadex G-25 column which was equilibrated and developed with o.I M Tris sulfate, and the eluate was freeze dried and dissolved in the requisite volume of ${ }^{2} \mathrm{H}_{2} \mathrm{O}$. The value of the ratio $A_{420} \mathrm{~nm} / A_{275} \mathrm{~nm}$ equalled 0.45 . The various spinach samples used for the ENDOR experiment were all adjusted to be $3.2-3.3 \mathrm{mM}$ in protein. They were transferred to matched EPR tubes, reduced with solid dithionite and frozen and stored in liquid nitrogen.

Parsley ferredoxin was isolated as previously described ${ }^{30}$. The apoprotein was prepared by successive precipitation with $8 \%$ trichloroacetic acid and dissolution in Tris- $\mathrm{HCl}$ buffer. Reconstitution was carried out under anaerobic conditions but otherwise under the same conditions as used for the same spinach protein. The resulting protein solution after concentration as described above was diluted 3 -fold with ${ }^{2} \mathrm{H}_{2} \mathrm{O}$ and freeze dried. Further removal of water was not attempted; the protein was 
dissolved in a small amount of ${ }^{2} \mathrm{H}_{2} \mathrm{O}$ and allowed to stand $\mathrm{I} .5 \mathrm{~h}$ at room temperature under nitrogen before reducing with solid sodium dithionite and freezing in liquid nitrogen. Samples thus obtained show a ratio, $A_{422 \mathrm{~nm}} / A_{277 \mathrm{~nm}}$ of $0.62-0.64$ and are identical in all measured properties to native protein.

Samples of putidaredoxin were prepared anaerobically by trichloroacetic acid precipitation and reconstitution with $\mathrm{FeCl}_{2}\left({ }^{56} \mathrm{Fe}\right.$ or $\left.{ }^{57} \mathrm{Fe}\right)$ and $\mathrm{Na}_{2} \mathrm{~S}^{31}$. Chemical analysis and biological activity of reconstituted samples were the same as with native proteins. Some samples were exchanged with ${ }^{2} \mathrm{H}_{2} \mathrm{O}$ by anaerobic dialysis at room temperature (final enrichment in ${ }^{2} \mathrm{H}$ is estimated to be $>90 \%$ ).

Pig adrenodoxin was isolated according to previously published procedures ${ }^{32}$ and the iron exchange was carried out as described by BEINERT AND ORME-JOHNSON ${ }^{\mathbf{3 3}}$.

\section{EXPERIMENTAL}

The products of these ${ }^{57} \mathrm{Fe}$ reconstitution experiments are indistinguishable from the native proteins by optical and CD spectroscopy and by activity measurements ${ }^{34}$, though the introduction of the ${ }^{57} \mathrm{Fe}$ does affect the EPR spectrum in the reduced state by means of the hyperfine interaction between the iron nuclei and the net electron spin. No extraneous iron can be detected in the reconstituted and purified proteins.

Spinach and parsley ferredoxin do not suffer any detectable deterioration on the one cycle of lyophilization ${ }^{26,30}$ used in the concentration procedure. The dithionitereduced proteins can be frozen and thawed several times without any measurable changes in the EPR spectra, and since the EPR spectrum is recognized as a physical assay for these proteins ${ }^{\mathbf{3 5}}$ this indicates that negligible deterioration results. For the experiments reported here the samples, once frozen in the reduced state were never allowed to thaw; thus the slight deterioration (approx. $5 \%$ ) which is known to occur on reoxidation ${ }^{36}$ was avoided.

\section{Electron-nuclear double resonance (ENDOR)}

ENDOR is a well-established technique for studying paramagnetic sites in single crystals ${ }^{37}$. In an imaginative series of experiments HYDE and co-workers extended this technique to the study of free radicals in solution ${ }^{38,39}$ and later to powdered samples containing paramagnetic ions ${ }^{40}$.

The method of ENDOR involves the detection of induced nuclear spin transitions by measuring the concomitant changes in the intensity of the EPR signals observed in the material of interest. The intensity of a partially saturated EPR spectrum is affected by applying to the sample a variable frequency radio-frequency field which can induce nuclear spin transitions. The detailed mechanism by which the nuclear transitions affect the EPR spectrum differs for various materials. To understand ENDOR is to understand at least one of these mechanisms; therefore, the reader is urged to consult the literature on this subject (see for example ref. 4I). A brief discussion here will serve as an introduction to the phenomenon.

For an EPR line whose width is mainly caused by random static interactions with neighboring spins (inhomogeneous broadening) the concept of a "spin packet" is often used. The overall line shape is the envelope of the multitude of spin packets, with a line width parameter $\Delta H$ (see Fig. I) which is not related to any relaxation 
process but rather depends upon the distribution of local static magnetic fields produced by the neighboring spins at the site in question. The individual "spin packet" is centered at an effective magnetic field $H=h v / g \beta$ with a line width $\delta H$ determined by a relaxation time $\tau_{2}\left(g \beta \delta H \sim \hbar / \tau_{2}\right)$ characteristic of processes which shorten the lifetime of the individual spin in any given quantum state and thus produce a broadening (called homogeneous broadening). These processes involve the high-frequency

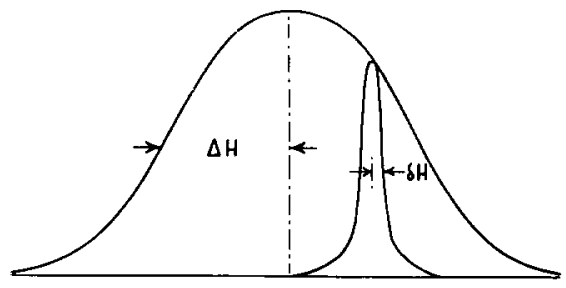

Fig. I. Spin packet inside the inhomogeneously broadened line $\left(g \beta \delta H \sim \hbar / \tau_{\mathbf{2}}\right)$.

(precessing) components of the magnetic fields of neighboring spins which set up an oscillatory field at the spin in question and induce magnetic resonance transtitions. In contrast, the static magnetic fields from these same spins do not shorten the lifetime but merely shift the location of the resonance absorption in a manner similar to that produced by the use of an inhomogeneous magnet - hence the name inhomogeneous broadening for the latter. Clearly such inhomogeneous broadening can obscure small hyperfine interactions which will thus go undetected in EPR; as we shall see, these can be resolved in ENDOR. The resulting line shape from such inhomogeneous broadening cannot be computed readily - a Gaussian distribution of field strengths is often used. Such inhomogeneous broadening is further compounded in the proteins studied here by the presence of " $g$ strain" i.e. the iron site of each protein has a slightly different conformation which results in a distribution of $g$ values and hence an apparent broadening of the EPR line which further obscures the hyperfine splittings. Such a Gaussian shape function has been found to give an acceptable fit to the EPR " $g$-strain" distribution and will be assumed in the computer simulated EPR and ENDOR spectra presented in this paper.

A second relaxation process of concern here is caused by the spin lattice interaction. The populations of the various spin states along the applied magnetic field are determined by interactions with the applied microwave or radiofrequency field and with the structure (lattice) in which the spin system is embedded. In the absence of any applied radiation, the spin populations will assume a Boltzmann distribution determined by the vibrational and rotational temperature of the lattice. In the case of a solid, the thermal motions consist of lattice vibrations and cause time varying oscillatory magnetic fields at the various spin sites by the relative motion with respect to neighboring magnetic moments. Such processes cause the spin state populations to approach the thermal equilibrium distribution at a characteristic mean time, $\tau_{\mathbf{1}}$, the spin lattice relaxation time. These transitions occur by a variety of processes which, in general, result in a $\tau_{1}$ which has a strong temperature dependence ${ }^{3}$ in the range from liquid-helium to room temperatures. This will be used to advantage in the ENDOR studies to follow.

To make the above more specific let us consider these relaxation paths and the 
phenomenon of ENDOR for a single unpaired electron $(S=1 / 2)$ interacting with a single nucleus $(I=1 / 2)$. For simplicity let us assume further that there is an isotropic hyperfine (Fermi contact) interaction between these two particles. The Hamiltonian describing this system in an applied static magnetic field $H$ is

$$
\begin{aligned}
\mathscr{H} & =-\vec{\mu}_{\mathrm{e}} \cdot \vec{H}-\vec{\mu}_{\mathrm{n}} \cdot \vec{H}+A \cdot \vec{I} \cdot \vec{S} \\
& =+g_{\mathrm{e}} \overrightarrow{\beta \mathrm{S}} \cdot \vec{H}-g_{\mathrm{n}} \beta_{\mathrm{n}} \vec{I} \cdot \vec{H}+A \cdot \vec{I} \cdot \vec{S}
\end{aligned}
$$

where $\mu_{\mathrm{e}}=-g_{\mathrm{e}} \overrightarrow{\beta S}$ is the magnetic moment of the electron; $\vec{\mu}_{\mathrm{n}}=g_{\mathrm{n}} \beta_{\mathrm{n}} \vec{I}$, the magnetic moment of the nucleus; $A$, the hyperfine constant; $\vec{I}$, the nuclear spin; $\vec{S}$, the electron spin; $g_{\mathrm{e}}$, the electron $g$ factor; $g_{\mathrm{n}}$, the nuclear $g$ factor; $\beta$, the Bohr magneton; $\beta_{\mathrm{n}}$, the nuclear magneton; and $H$, the applied magnetic field. The energies of this system are plotted in Fig. 2a as a function of the applied magnetic field $H$. In high field these are given by

$$
E=+g_{\mathrm{e}} \beta m_{\mathrm{s}} H-g_{\mathrm{n}} \beta m_{\mathrm{I}} H+A m_{\mathrm{l}} m_{\mathrm{s}}
$$

It is convenient to display the four energy levels existing at some large fixed applied field, $H_{0}$, in the manner of Fig. $2 \mathrm{~b}$, where each level is labeled at the side by the electron spin quantum number, $m_{\mathrm{s}}$, and above by the nuclear quantum number, $m_{\mathrm{I}}$. It is assumed that $A \gg g_{\mathrm{n}} \beta_{\mathrm{n}} H$ which is the case in these studies. The dotted lines

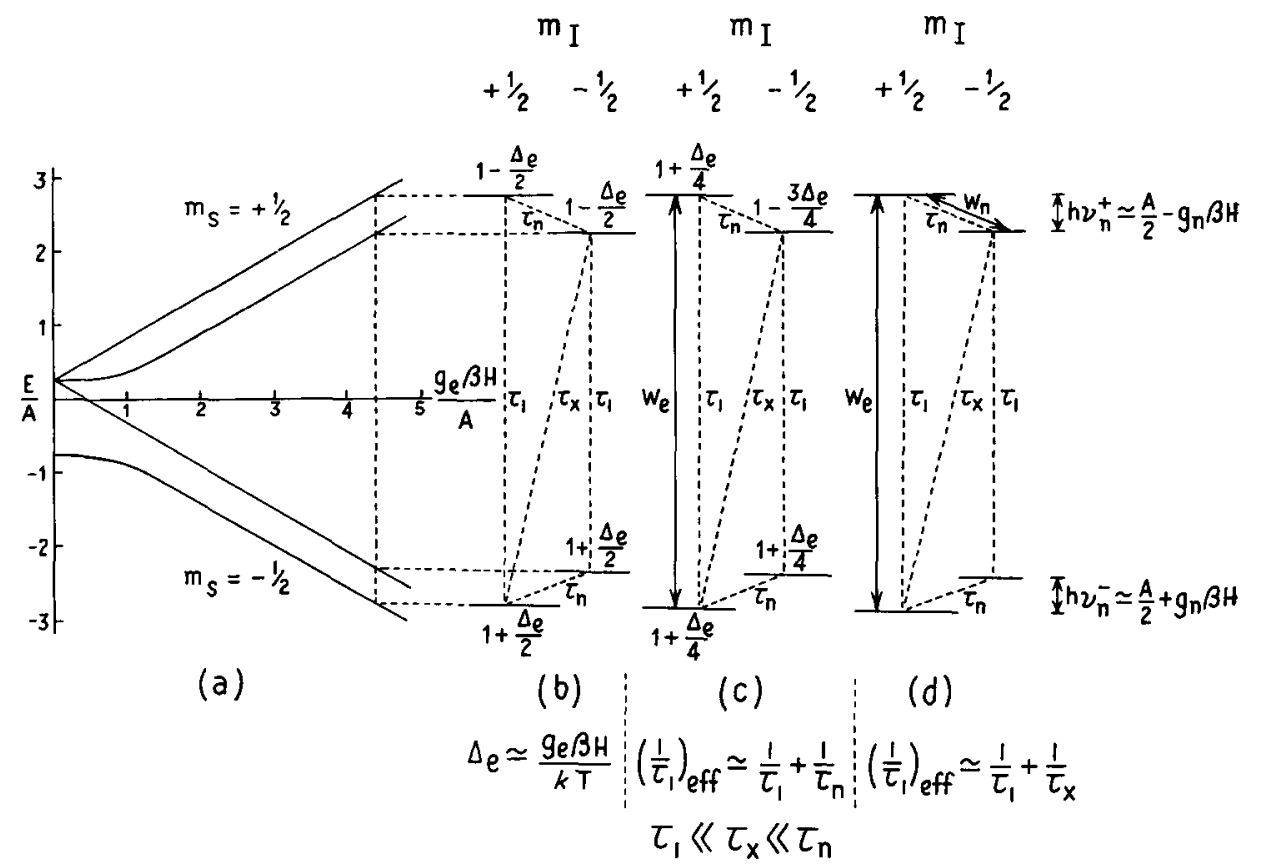

Fig. 2. (a) The energy level diagram as a function of $H$ for an $S=1 / 2, I=1 / 2$ system with $I \cdot S$ coupling $\left(A \gg g_{\mathrm{n}} \beta_{\mathrm{n}} H\right)$. (b) The energy levels, relaxation paths and approximate Boltzmann populations at a fixed applied magnetic field. (c) The same as (b) but with the $\Delta m_{\mathrm{s}}=\frac{1}{-1}, m_{\mathrm{I}}=$ $+1 / 2$ transition induced at a rate $W_{\mathrm{e}} \gg \mathrm{I} / \tau_{1}$. (d) The same as (c) but with the $A m_{\mathrm{I}}=-1, \mathrm{r}, m_{\mathrm{s}}=+$ $1 / 2$ transition induced at a rate $W_{\mathrm{n}}>\mathrm{I} / \boldsymbol{\tau}_{\mathbf{x}}$. 
in Fig. 2 b denote the principal relaxation paths (there are others), and the approximate thermal equilibrium population in each level is noted above the level. The relaxation rate at which direct nuclear transitions occur is denoted by $\mathrm{I} / \tau_{\mathrm{n}}$, the rate at which electron transitions occur by $I / \tau_{\mathbf{1}}$ and the rate at which cross-relaxation occurs by $\mathrm{I} / \tau_{\mathbf{x}}$. Most generally, $\tau_{1} \leqslant \tau_{\mathbf{x}} \leqslant \tau_{\mathrm{n}}{ }^{37}$.

From Fig. $2 \mathrm{~b}$ it may be seen that inducing nuclear transitions $\left(\Delta m_{\mathrm{I}}= \pm_{\mathrm{I}}\right.$, $\left.\Delta m_{\mathrm{s}}=0\right)$ at $h \nu_{\mathrm{n}}{ }^{ \pm}$will have a very small effect on the populations of these levels since the population ratio differs from unity initially by only half the nuclear hyperfine constant divided by $k T\left(\Delta_{\mathrm{e}}=A / 2 k T\right)$, i.e. the Boltzmann population ratio is small because $A \ll \mathrm{g}_{\mathrm{e}} \beta H$. On the other hand, if one of the electronic transitions $\left(\Delta m_{\mathrm{s}}= \pm \mathbf{I}\right.$, $\Delta m_{\mathrm{I}}=o$ ) is saturated by exposing the sample to high microwave power the populations of the affected levels will be equalized (with the remainder taking on a Boltzmann distribution). The steady-state populations are those in Fig. $2 \mathrm{c}$ and the population ratio between the nuclear states $\left(m_{\mathrm{s}}=+1 / 2, m_{1}= \pm 1 / 2\right)$ is now much larger. If the nuclear transition $h \nu_{\mathbf{n}}{ }^{+}$is now induced rapidly compared to $I / \tau_{\mathbf{x}}$, the population of the $m_{\mathrm{s}}=+1 / 2, m_{\mathrm{I}}=+1 / 2$ state is strongly affected because of the initial difference in the populations of the $m_{\mathrm{s}}=+1 / 2, m_{\mathrm{I}}=+1 / 2$ and the $m_{\mathrm{s}}=+1 / 2, m_{\mathrm{I}}=-1 / 2$ states which are now equalized by the saturating nuclear transistions. This results in a pronounced increase in the power absorbed in the electronic transition $\left(\Delta m_{\mathrm{s}}= \pm \mathrm{I}\right.$, $\left.m_{1}=+1 / 2\right)$ because the electronic difference population is now increased by the nuclear transition. (The power absorbed is proportional to the product of three terms: the population difference, the energy quantum and the transition probabilities.) Thus the presence of the nuclear transition can be detected by observing a change in the power absorbed in the electron transition which is considerably larger than that absorbed in the nuclear transition itself - this is the phenomenon of ENDOR as it is effected by the creation of population differences. This process of changing the relative population differences of the two electron states by the flipping of nuclear spins is called "packet shifting".

Another effect of the combined electron and nuclear transitions can be seen by the change wrought in the apparent electron relaxation time.

Fig. 2c shows that for the EPR transition noted $\left(m_{\mathrm{I}}=+1 / 2\right)$ the effective relaxation rate between these two levels is determined primarily by the direct path $\left(\mathrm{I} / \tau_{1}\right)$. This is shunted by the indirect path via the $\left(m_{\mathrm{s}}=+1 / 2, m_{\mathrm{x}}=-1 / 2\right)$ state which has an effective rate approximately $\mathrm{I} / \tau_{\mathrm{n}}$ since $\tau_{\mathrm{n}} \geqslant \tau_{\mathrm{x}}$ and hence the slower process $\left(I / \tau_{n}\right)$ controls the rate via this indirect path. The total effective relaxation between the electronic states of Fig. $2 \mathrm{c}$ is thus $\mathrm{I} / \tau_{1}+\mathrm{I} / \tau_{\mathrm{n}}$. If on the other hand, the nuclear transition is induced instead as in IFig. $2 \mathrm{~d}$, the effective relaxation rate between the nuclear states involved is by the direct path $\left(I / \tau_{\mathrm{n}}\right)$ shunted by a much more effective indirect path via the $\left(m_{\mathrm{s}}=+1 / 2, m_{\mathrm{I}}=--1 / 2\right)$ state at a rate approximately $I / \tau_{\mathbf{x}}$ (since $\tau_{\mathbf{x}} \gg \tau_{1}$ ). The effective relaxation between muclear levels is thus $I / \tau_{n}+I / \tau_{x}$ which is dominated by $I / \tau_{x}$; thus, if the nuclear transition is to be effective in changing the populations of the states it must be induced at a rate $W_{\mathrm{n}}$ such that $W_{\mathrm{n}} \tau_{\mathrm{x}} \geqslant \mathrm{I}$ (not just $W_{\mathrm{n}} \tau_{\mathrm{n}} \geqslant \mathrm{I}$ ). Notice that the inducing of the electronic transition does not change this condition. However, the inducing of the nuclear transition does change the effective relaxation between the electronic levels since it influences the relaxation rate via the indirect path; if $W_{\mathrm{n}} \gg \mathrm{I} / \tau_{\mathbf{x}}$ then the electronic relaxation rate via the indirect path is determined by $\mathrm{I} / \tau_{\mathbf{x}}$ (instead of $\mathrm{I} / \tau_{\mathrm{n}}$ as in Fig. $2 \mathrm{c}$ ). The 
application of the nuclear resonance thus changes the effective electronic relaxation rate from $I / \tau_{1}+I / \tau_{\mathbf{n}}$ (Fig. 2 ) ) to $I / \tau_{1}+I / \tau_{\mathbf{x}}$ (Fig. $2 \mathrm{~d}$ ); if the EPR signal is partially saturated, this change in the electronic relaxation rate will manifest itself as a change in the EPR signal amplitude. Thus the presence of the nuclear resonance absorption may be detected by monitoring the saturated EPR signal - this is again the phenomenon of ENDOR but effected by the change made by the nuclear transition in the apparent electron relaxation time.

We thus see two different mechanisms for the ENDOR process: (a) "packet shifting" and (b) a change in the apparent relaxation rates. There is still another mechanism called "distant ENDOR" whereby nuclear transitions induced in nuclei which are suffciently far away from the unpaired electron as not to have any direct hyperfine coupling may perturb the electron states by the process of spin diffusion. Because this mechanism is of no direct importance for the iron ENDOR signals discussed in this paper, no further mention of this third mechanism will occur.

\section{Anisotropic g-and A-tensors}

It is to be noted that for our simple model displayed in Fig. 2, the inducing of the second nuclear transition $\left(h v_{\mathrm{n}}{ }^{-}\right)$has a much smaller effect on the effective electronic relaxation time for the EPR transition chosen. In fact, $\delta \tau_{1} / \tau_{1} \simeq-\tau_{1} / \tau_{\mathrm{n}}$ for this case which is a much smaller change than that produced by the transition $h n_{n}{ }^{+}$, so small that it probably would not be detected.

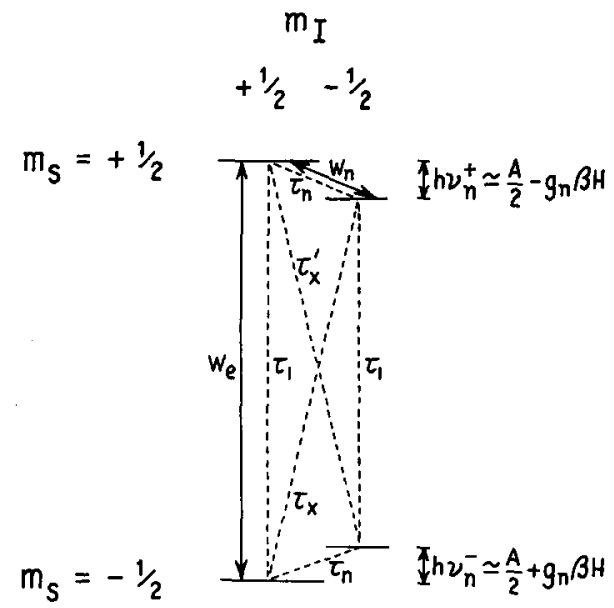

$$
\begin{aligned}
& \text { For } h v_{n}^{+}:\left(\frac{1}{\tau_{1}}\right)_{\text {eff }} \simeq \frac{1}{\tau_{1}}+\frac{1}{\tau_{x}} \\
& \text { For h } v_{n}^{-}:\left(\frac{1}{\tau_{1}}\right)_{\text {eff }} \simeq \frac{1}{\tau_{1}}+\frac{1}{\tau_{x}^{\prime}}
\end{aligned}
$$

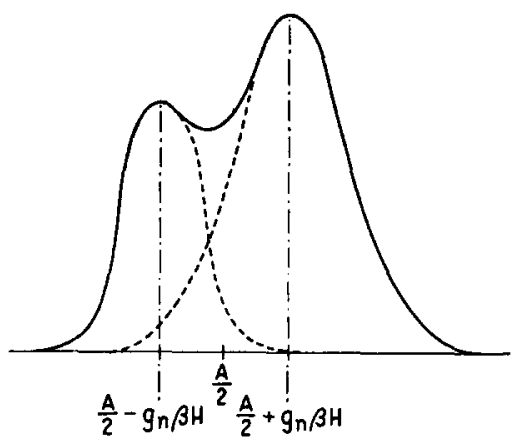

Fig. 3. The energy level diagram for an $S=1 / 2, I=1 / 2$ system in a fixed magnetic field with anisotropic hyperfine coupling and the $\Delta m_{\mathrm{B}}= \pm \mathrm{I}, m_{\mathrm{I}}=+1 / \mathrm{s}$ transition induced at the rate $W_{\mathrm{e}} \gg$ $\mathrm{I} / r_{1}$ and either the $\Delta m_{\mathrm{I}}= \pm \mathrm{I}, m_{\mathrm{g}}=+1 / 2$ or the $\Delta m_{\mathrm{I}}= \pm \mathrm{I}, m_{\mathrm{g}}=-1 / 2$ transition induced at $W_{\mathrm{n}} \geqslant \mathrm{I} / \tau_{\mathrm{x}}$ or $\mathrm{r} / \boldsymbol{\tau}_{\mathrm{x}}{ }^{\prime}$.

Fig. 4. A predicted ${ }^{57} \mathrm{Fe}$ ENDOR spectrum for a single orientation. 
A more general model can be obtained by allowing the $g$-and $A$-tensors to be anisotropic. When the hyperfine interaction is anisotropic, transitions between the $m_{\mathrm{s}}=+1 / 2, m_{\mathrm{I}}=+1 / 2$ and $m_{\mathrm{s}}=-1 / 2, m_{\mathrm{I}}=-1 / 2$ states are allowed and a new relaxation path is created with a characteristic relaxation time $\tau_{\mathbf{x}}{ }^{\prime}$ as shown in Fig. 3 . This relaxation rate depends on the degree of anisotropy. Since the two nuclear transitions now result in fractional changes in the effective electronic relaxation times of $\delta \tau_{1} / \tau_{1} \simeq-\tau_{1} / \tau_{\mathbf{x}}$ and $\delta \tau_{1} / \tau_{1} \simeq-\tau_{1} / \tau_{\mathbf{x}}{ }^{\prime}$, respectively, the resulting ENDOR signals will not be equal in general. With the above in mind, a hypothetical ENDOR spectrum may be constructed using Eqn. 2 to obtain the nuclear transition frequencies as follows:

$$
\begin{aligned}
h \nu_{\mathrm{n}}^{ \pm} & =\left|\Delta E\left(m_{\mathrm{s}}= \pm \mathbf{I} / 2\right)\right| \\
& =\left|E\left(m_{\mathrm{s}}= \pm \mathrm{r} / 2, m_{\mathrm{I}}=+\mathrm{I} / 2\right)-E\left(m_{\mathrm{s}}= \pm \mathrm{I} / 2, m_{\mathbf{I}}=-\mathbf{I} / 2\right)\right| \\
& =\left|\left( \pm \frac{g_{\mathrm{e}} \beta H}{2}-\frac{g_{\mathrm{n}} \beta H}{2} \pm \frac{A}{4}\right)-\left( \pm \frac{g_{\mathrm{e}} \beta H}{2}+\frac{g_{\mathrm{n}} \beta H}{2} \mp \frac{A}{4}\right)\right| \\
& =\left|-g_{\mathrm{n}} \beta H \pm \frac{A}{2}\right|
\end{aligned}
$$

which shows that two lines will be observed corresponding to the two values of $m_{1}$ and they will be centered at frequencies given by the absolute values of Eqn. 3 . For protons not directly bonded to the paramagnetic center, $g_{\mathrm{n}} \beta H>A / 2$ and a pair of lines centered at $g_{\mathrm{n}} \beta H$ and separated by A will be observed. For ${ }^{57} \mathrm{Fe}$ in the nucleus of the paramagnetic site, $A / 2 \geqslant g_{\mathrm{n}} \beta H$ and a pair of lines centered at $A / 2$ and separated by $2 g_{\mathrm{n}} \beta H$ will result. Fig. 4 displays this latter case for a realistic set of parameters for one of the iron sites in the two-iron ferredoxins at $H_{o}=3200$ Gauss where

$$
\frac{g_{\mathrm{n}} \beta H_{0}}{h} \simeq 0.45 \mathrm{MHz} \text { and } \frac{A}{2 h} \simeq 2 \mathrm{I} \mathrm{MHz}
$$

In the systems that have anisotropic $g$ - and $A$-tensors, the spectra would look exactly like Fig. 4 for any given molecular orientation; however, for a frozen solution of protein molecules, all orientations are present and the spectra are complicated by this superposition of spectra from a variety of orientations. Not all orientations contribute to the ENDOR spectra, because the $g$-tensor is anisotropic and hence not all orientations are undergoing EPR. By selecting the magnetic field one may select molecules of various orientations to undergo EPR and hence ENDOR; thus it is possible to obtain the values of the components of the effective $A$-tensor along the principal axes directions of the $g$-tensor. It will be shown that it is possible to decide from the nature of the resulting ENDOR spectra whether or not the $A$-tensor principal axes are coincident with the g-tensor principal axes. If they are coincident it is trivially simple to obtain the $A$-tensor principal axis values directly from the ENDOR spectra of the amorphous sample without the need for obtaining single crystals of the protein. If the axes are not coincident, the task of obtaining the principal axis $A$-values is more complicated and a computer program is required. 


\section{EPR spectral synthesis}

The synthesis of EPR spectra for amorphous samples has been discussed by several authors ${ }^{42-47}$ (see also the references in ref. 48). In brief review, for spin 1 systems the resonance condition is given by

$$
h v_{0}=g \beta H
$$

where $v_{0}$ is the applied microwave frequency, $g$ is the electron $g$-factor, $\beta$ is the Bohr magneton and $H$ is the applied magnetic field. For a magnetic field applied at the Euler angles $\beta, \gamma$ with respect to the molecular $g$-tensor principal axes as shown in Fig. 5 .

$$
g=\left(g x^{2} \sin ^{2} \beta \cos ^{2} \gamma+g y \sin ^{2} \beta \sin ^{2} \gamma+g z^{2} \cos ^{2} \beta\right)^{1 / 2}
$$

If all molecular orientations are equally likely then the number of molecules having a magnetic field oriented at angles between $\beta$ and $\beta+\mathrm{d} \beta, \gamma$ and $\gamma+\mathrm{d} \gamma$, is given by the solid angle at these orientations; i.e.

$$
\mathrm{d} N=N_{\mathbf{o}} \sin \beta \mathrm{d} \beta \mathrm{d} \gamma
$$

Eqns. 4 and 5 may be solved for the magnetic field values at which these molecules undergo resonance absorption. The number of molecules absorbing at magnetic field values between $H$ and $H+\mathrm{d} H$ is given by $\mathrm{d} N / \mathrm{d} H$. An analytical expression may be obtained from Eqns. 4 and 5 relating $\sin \beta \mathrm{d} \beta \mathrm{d} \gamma$ to $H$ and $\mathrm{d} H$. These expressions are given by several authors ${ }^{42-46}$ and are plotted versus applied magnetic field as dotted lines in Fig. 6 for a specific set of parameters.
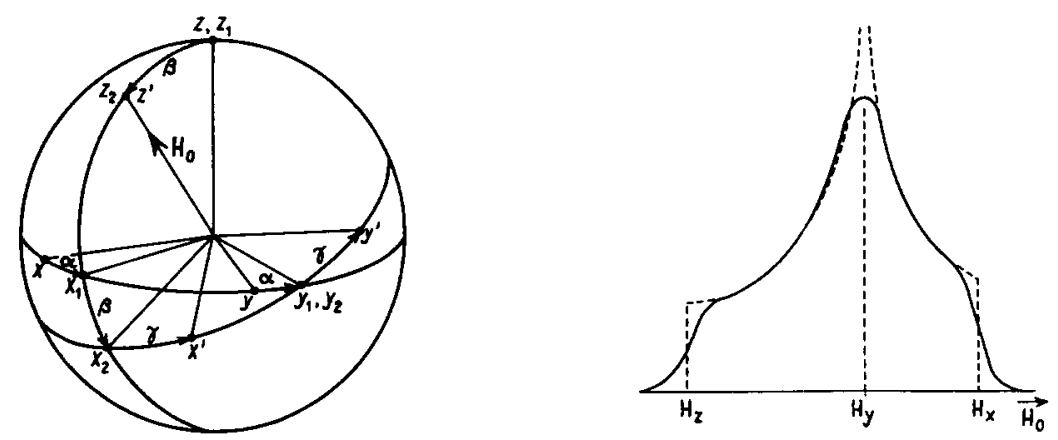

Fig. 5. The orientation of the applied magnetic field, $H_{\mathbf{0}}$, with respect to the $g$-tensor principal axes on the molecule in terms of Euler angles $\beta$ and $\%$

Fig. 6. A calculated integrated EPR absorption spectrum resulting from an amorphous sample with $H_{z}<H_{y}<H_{x}$ where $H_{i}=h v_{0} / g_{i} \beta$. The dotted lines represent $\mathrm{d} N / \mathrm{d} H$, the number of molecules with an absorption center occuring between $H$ and $H+\mathrm{d} H$, and the solid curve is the actual absorption curve resulting from a Gaussian absorption spectrum for each molecule.

Notice that $H_{x}=h v_{0} / g_{x} \beta$ is the magnetic field value at which molecules having their $g$-tensor $x$-axes collinear with the applied field will absorb microwave power, i.e. undergo EPR. Likewise, $H_{z}=h v_{\mathrm{o}} / g_{z} \beta$ is the magnetic field value at which molecules having their $g$-tensor $z$-axes collinear with the applied field will absorb; since $H_{z}<\mathrm{H}_{y}<\mathrm{H}_{x}$ by assumption, $H_{y}=h v_{\mathrm{o}} / g_{y} \beta$ is the magnetic field where not only molecules with their $y$-axes aligned collinear with the field will absorb but also mole- 
cules of a large number of other orientations (such that $g=g y$ for those orientations) will absorb. This is the reason for the much greater intensity at $H_{y}$.

To obtain the actual absorption spectrum it is necessary to plot the spectral intensity versus magnetic field where the absorption line shape for each orientation is multiplied by the transition probability at that orientation and the number of molecules having that orientation. This is most easily accomplished by use of a computer in the cases where the transition probabilities or line shapes differ markedly with orientation. Furthermore, when one or more hyperfine interactions are present a computer simulation is almost a necessity. No detailed description of our EPR program will be given because computer algorithms to fit EPR spectra are now abundant in the literature ${ }^{42-47}$ (see also the references in ref. 48 ). If one includes the hyperfine interactions from two non-equivalent iron atoms but treats the ligand hyperfine and "g-strain" by using a Gaussian line shape of variable width it is possible to obtain a reasonably good fit to all of the ferredoxin spectra. This is of some importance because the resulting ENDOR spectral synthesis is sensitive to the EPR lineshape. The ENDOR spectral synthesis is used to extract the $A$-tensor principal axis values from the ENDOR data; hence the lineshape fit to the EPR data is essential. Fig. 7 shows the EPR computer fit for adrenodoxin. This fit employs the parameters obtained from the ENDOR studies (Table I) and will be discussed later.

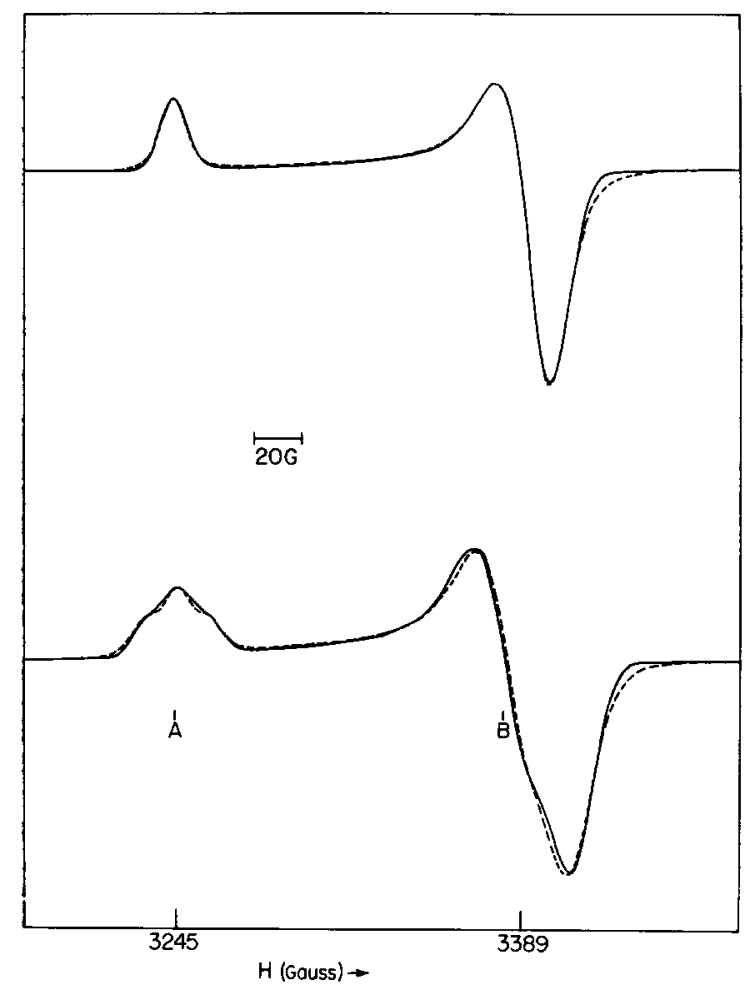

Fig. 7. The experimental (--) and synthesized (- - E) EPR spectra for reduced (top) ${ }^{56} \mathrm{Fe}$ and (bottom) $91.5 \%$ enriched ${ }^{57} \mathrm{Fe}$ reconstituted adrenodoxin. The calculated spectra result from the parameters in Table I and Gaussian line shapes of widths 8.I, 9.4 and 5.6 Gauss with the field along the $x, y$ and $z$-axes, respectively. 


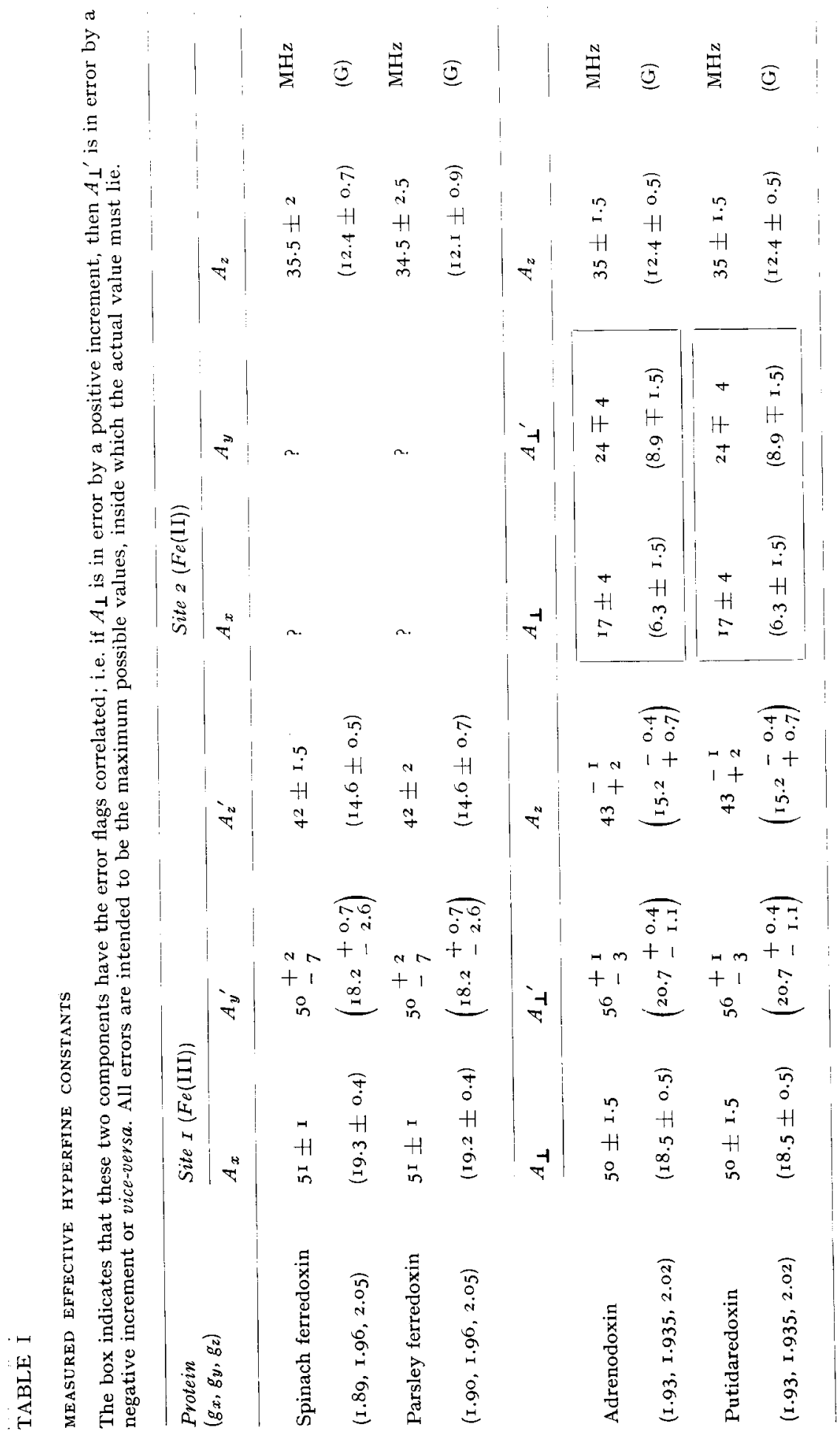

Biochim. Biophys. Acta, 253 (I97 I) IIo-I 33 


\section{ENDOR from amorphous samples}

It is possible to use the anisotropy in the electron $g$-factor to select molecules of a given set of orientations to undergo $\operatorname{ENDOR}^{49}$; a brief discussion follows of how this may be done.

The comparison of Figs. 5 and 6 shows that by setting the magnetic field at some value between $H_{x}$ and $H_{z}$, only those molecules having a specific set of orientations will be undergoing EPR. By choosing this field, the set of orientations may be chosen; e.g. if the magnetic field is set below $H_{z}$ (assuming $H_{x}>H_{y}>H_{\alpha}$ ) then primarily only those molecules whose $z$-axes are aligned along the applied field will be undergoing EPR. Similarly, if the field is set above $H_{x}$, primarily those molecules whose $x$-axes are aligned along the field will be undergoing EPR; if we set the field at $H_{y}$ (between $H_{x}$ and $H_{z}$ ) molecules of many orientations will be contributing to the EPR spectrum in addition to those molecules whose $y$-axes are along the applied field. BY keeping track of which molecular orientations are contributing to the EPR and hence ENDOR it is possible to simulate the ENDOR spectrum and by comparison with the experimental spectrum to obtain the components of the $A$-tensor. The details of the ENDOR spectral synthesis employed here will be published elsewhere ${ }^{50}$.

\section{Instrumentation}

The basic ENDOR instrument is nearly the same as that described by Hrlle AND MAKI ${ }^{38}$ and $\mathrm{HYDE}^{39}$, and the reader is referred to these papers for details. The one major difference is that the magnetic field modulation (at $47 \mathrm{~Hz}$ ) is approximately square-wave rather than sinusoidal. This was an essential change which results in an ENDOR spectrum from only one magnetic field setting (the other being chosen off the EPR resonance absorption when possible). When the EPR signal is isotropic as in the case of free radicals in solution as studied by HYDE AND $\mathrm{MAKI}^{38}$ and HYDE ${ }^{39}$, the sinusoidal modulation presents no problem; however, when the EPR spectrum is anisotropic, the sinusoidal modulation yields ENDOR spectra from a continuum of magnetic field values and hence molecular orientations and thereby greatly complicates the spectral interpretation.

In brief, the instrument consists of an X-band homodyne EPR bridge with a separate bias arm to permit linear operation from $3 \mu \mathrm{W}$ to $300 \mathrm{~mW}$ incident on a Varian Model V4533-Cylindrical EPR Cavity operating in the $\mathrm{TE}_{\mathbf{0 1 1}}$ mode. The sample is placed in a $3-\mathrm{mm}$ internal diameter, $4.3-\mathrm{mm}$ outer diameter quartz tube which rests inside a quartz dewar insert. The radio-frequency field to induce the nuclear transitions is applied perpendicular to the d.c. applied field by coils external to the microwave cavity. The helical wound side walls of the cavity permit the radio frequency field to penetrate to the sample. To avoid serious overheating of the microwave cavity this radio-frequency field is applied as a pulse train. The pulse width is I $8 \mu \mathrm{sec}$ and the pulse repetition rate is $6 \mathrm{kHz}$. Alternate pulses are at slightly different frequencies, $f$ and $f+\Delta f$, to permit automatic tracking of a voltage variable oscillator to the mechanically swept amplifiers. The frequency modulation, $A f$, is chosen to be smaller than the ENDOR linewidths so that it produces a negligible effect as far as ENDOR is concerned. All spectra displayed in this paper were taken with $\lambda f<20 \mathrm{kHz}$.

The power amplifiers contain four tuned circuits. Three of these are tracked and mechanically swept by a stepping motor which is pulsed at rates yielding appropriate frequency sweep rates (usually $3^{\circ} \mathrm{kHz} / \mathrm{sec}$ when using I-sec integrating times). The 
fourth tuned circuit is in the final output stage (cavity coils and vacuum variable capacitor) and is tracked by a motor deriving its drive from a phase detector monitoring the $3-\mathrm{kHz}$ component of the rectified output, thereby forcing the final stage to tune midway between $f$ and $f+\Delta f$ at all times. For the spectra displayed in this paper, the rotating radio-frequency field amplitude was typically 5 Gauss and this was held constant across the frequency scan by manual adjustment of the plate voltage on the final power stage.

The output from the EPR bridge is amplified and serially phase detected at 6 $\mathrm{kHz}$ and $47 \mathrm{~Hz}$ as discussed by $\mathrm{HYDE}$ AND MAKI ${ }^{38}$ and $\mathrm{HYDE}^{39}$. This results in an integral ENDOR signal display. The amplitude of the square-wave magnetic field modulation is typically 60 to 120 Gauss. The output of the $47-\mathrm{Hz}$ phase detector is displayed on the $y$-axis of a $X-Y$ recorder. The $X$-axis of the recorder is swept coherently with the variable frequency oscillator by a digital-to-analog converter which is driven in turn by a digital frequency meter tracking the oscillator.

\section{RESLLTS}

The EPR spectrum of the two-iron ferredoxin from pig adrenal cortex enriched with ${ }^{57} \mathrm{Fe}$ and diluted in ${ }^{2} \mathrm{H}_{2} \mathrm{O}$ is displayed in Fig. 7. Notice the $\mathrm{I}: 2: \mathrm{I}$ hyperfine structure around $H_{z}$ which has previously been interpreted as showing the near equality of the two iron $A$-values ${ }^{32}$. This latter interpretation will be examined later in the light of the ENDOR results. The synthesized spectra do not match perfectly with the experimental spectra - this is presumably caused by the use of Gaussian line shapes which is incorrect as can be seen by comparing the calculated and experimental spectra in the wings. No attempt was made to obtain a better line shape function.

When the magnetic field and square-wave modulation are set at the condition for detecting those molecules whose g-tensor $z$-axes are along the applied magnetic field (position A, Fig. 7) the ENDOR spectrum displayed in Fig. 8a is obtained. The signals from 8 to I9 $\mathrm{MHz}$ occur in pairs (of unequal intensity) centered around I3.5 $\mathrm{MHz}$ which is the free proton frequency. This characterizes these signals as being due to protons. Since they persist in ${ }^{2} \mathrm{H}_{2} \mathrm{O}$ solution they presumably arise from nonexchangeable protons on the protein located near the iron site - - these resonances will be the subject of a later communication. Between 20 and $25 \mathrm{MHz}$ is an ENDOR absorption showing a doublet separated by approximately $0.9 \mathrm{MHz}$ and centered at 2I.5 $\mathrm{MHz}$ together with a long absorption tail extending to higher frequencies. This separation is just twice the ${ }^{57} \mathrm{Fe}$ free NMR frequency - this fact plus the additional vanishing of this signal in the ${ }^{56} \mathrm{Fe}$ reconstituted protein ( $c f$. Fig. ga) establishes this signal as due to ${ }^{57} \mathrm{Fe}$.

When the magnetic field is increased to $H_{\perp}=h v_{\mathrm{o}} / g_{\perp} \beta$ (position B, Fig. 7) to detect molecules where the applied magnetic field is in the $x-y$ plane of the $g$-tensor principal axis system, the ENDOR spectrum of Fig. $8 \mathrm{~b}$ is obtained. Here again the signals occurring between 8 and $20 \mathrm{MHz}$ and centered around $\mathrm{I}_{4} \mathrm{MHz}$ (the new free proton NMR frequency in this higher magnetic field) may be interpreted as due to protons. The absorption between 23 and $29 \mathrm{MH}$ may be attributed to iron ( $c f$. Fig. 9 b). Notice that these are at higher frequencies than the signals attributed to iron at $H_{z}$ (Fig. 8a). In fact, the position of these signals may be followed as the field is incremented and hence the sampled molecules made to pass continuously from those for 
which the field is along the $z$-axis of the molecules g-tensor to those for which the field is in the $x-y$ plane.

Fig. 9 is an enlargement of the iron ENDOR region and shows a comparison of the ENDOR spectra obtained from pig adrenodoxin with ${ }^{57} \mathrm{Fe}$ and ${ }^{56} \mathrm{Fe}$ respectively, substituted in the iron site. It is clear from a comparison of these spectra that the ENDOR signals in the ${ }^{57} \mathrm{Fe}(I=1 / 2)$ sample are absent in the ${ }^{56} \mathrm{Fe}(I=0)$ sample, thus positively identifying them as due to ${ }^{57} \mathrm{Fe}$.

$\mathbf{a}$
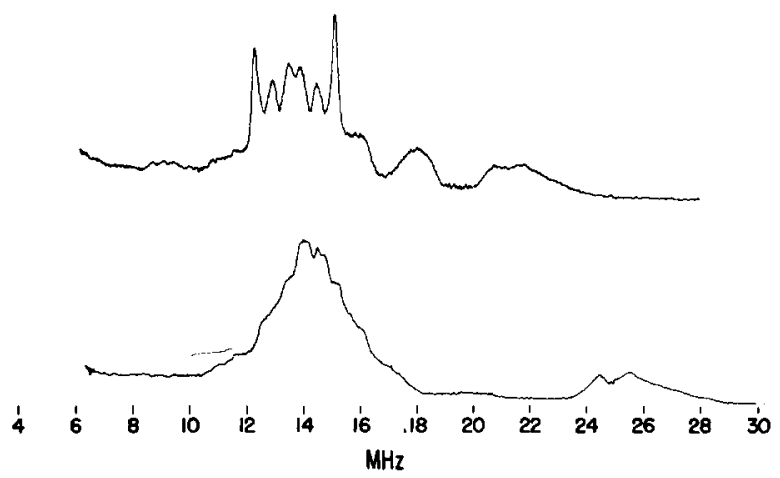
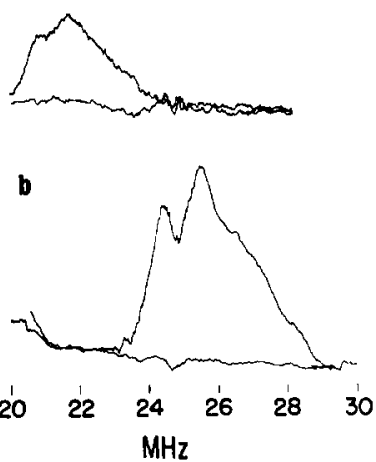

Fig. 8. The ENDOR spectrum observed for reduced adrenodoxin in ${ }^{2} \mathrm{H}_{2} \mathrm{O}$ at $\mathrm{I} 9 \mathrm{~K}$ and (top) with the applied field set at position A, Fig. 7 , so as to select molecules with the $z$-axes aligned along the magnetic field and (bottom) with the magnetic field set at position B, Fig. 7, so as to select molecules with orientations such that the magnetic field is somewhere in the $x-y$-plane of the molccular $g$-tensor principal axis system.

Fig. 9. The ENDOR spectra at $19 \mathrm{~K}$ for reduced adrenodoxin in ${ }^{2} \mathrm{H}_{2} \mathrm{O}$, reconstituted with $57 \mathrm{Fe}$ (upper trace) or ${ }^{56} \mathrm{Fe}$ (lower trace) with the magnetic field set at (a) $H_{z}$ and (b) $H_{\perp}$

When the ENDOR signals occurring between Io and $20 \mathrm{MHz}(c f$. Fig. 8) for the ${ }^{57} \mathrm{Fe}$ and ${ }^{56} \mathrm{Fe}$ substituted proteins are carefully compared at lower temperatures and higher microwave powers there are small but real differences observed. These are shown in Fig. Io. All difference spectra are obtained by slightly adjusting the spectrometer gains to give equal EPR intensities for the ${ }^{57} \mathrm{Fe}$ and ${ }^{56} \mathrm{Fe}$ EPR spectra, respectively. The ENDOR baselines were set equal at frequencies where no ENDOR was expected. Fig. Ioa displays the spectra obtained when the molecules whose $z$-axes are aligned along the magnetic field are selected for observation by appropriate choice of the applied field. Note the clearly discernible difference in intensity centered at I7.5 $\mathrm{MHz}$ in addition to the low-frequency portion of the previously identified ${ }^{57} \mathrm{Fe}$ ENDOR signal at 2I MHz. This new signal has a width approximately equal to twice the ${ }^{57} \mathrm{Fe}$ NMR frequency and is apparently attributable to ${ }^{57} \mathrm{Fe}$ also. In addition, there is a smaller but reproducible difference centered around I3 $\mathrm{MHz}$ which must be suspected as being due to ${ }^{57} \mathrm{Fe}$. To test conclusively that these difference signals are direct ${ }^{57} \mathrm{Fe}$ ENDOR signals it was necessary to change the microwave resonant frequency of the cavity and thus the value of the applied magnetic field at which the free proton NMR occurred. If the signals moved with the applied field they were apparently due to protons whose $\tau_{1}$ was affected by the ${ }^{57} \mathrm{Fe}$ substitution and if they stayed at the same ENDOR frequency they could be properly interpreted as ${ }^{57} \mathrm{Fe}$ ENDOR signals from another iron site in the protein. Fig. II displays the result obtained from this 


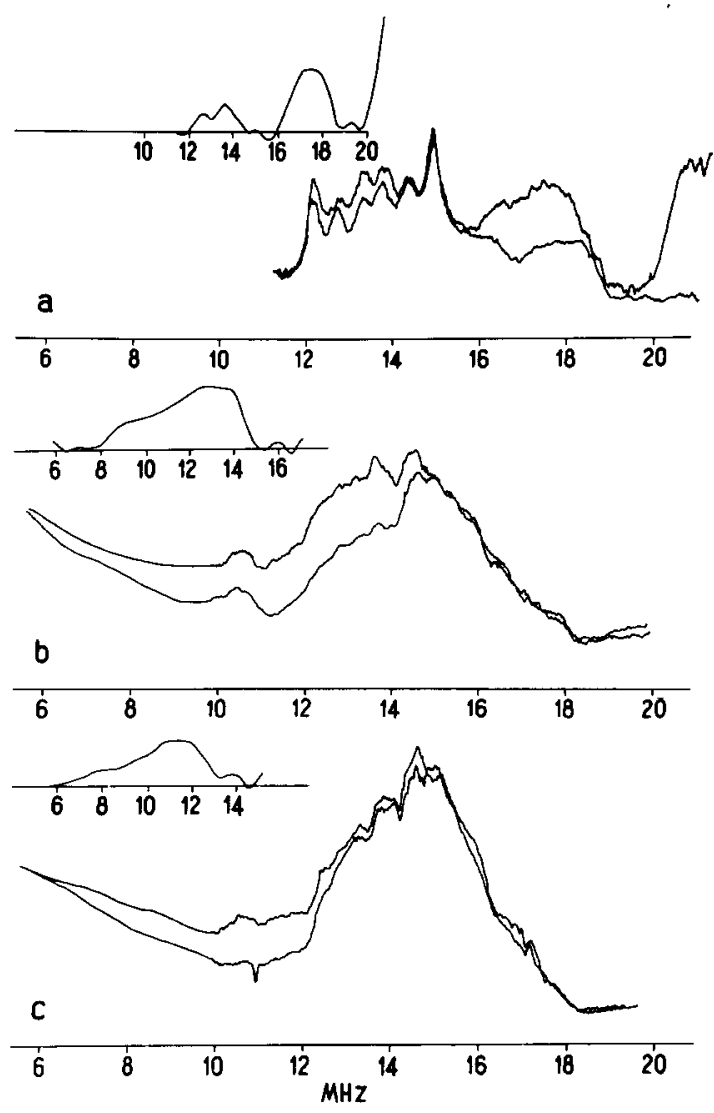

Iig. 3o. The ENDOR spectra at $13{ }^{\circ} \mathrm{K}$ and $v_{0}=9250 \mathrm{MHz}$ for reduced adrenodoxin in ${ }^{2} \mathrm{H}_{2} \mathrm{O}$ reconstituted with ${ }^{57} \mathrm{Fe}$ (upper trace) or ${ }^{56} \mathrm{Fe}$ (lower trace) with the magnetic field set at (a) $H_{z}$, (b) $H_{\perp}-16$ gauss and (c) $H_{\perp}$. The difference spectrum at each field setting is plotted on a reduced scale in the upper left.

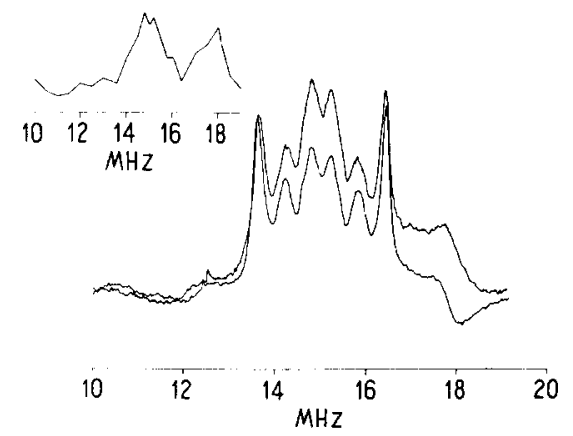

Fig. I . The ENDOR spectra at $13^{\circ} \mathrm{K}$ and $v_{0}=10$ ooo $\mathrm{MHz}$ for reduced adrenodoxin in ${ }^{2} \mathrm{H}_{2} \mathrm{O}$, reconstituted with ${ }^{57} \mathrm{Fe}$ (upper trace) or ${ }^{56} \mathrm{Fe}$ (lower trace) with the magnetic field set at $H_{z}$. The difterence spectrum is plotted every $0.5 \mathrm{MHz}$ on a reduced scale in the upper left. This is to he compared with the spectra in Fig. roa. 
experiment. Notice that the difference spectrum at $17.5 \mathrm{MHz}$ remains stationary and is thus attributable to a second ${ }^{57} \mathrm{Fe}$ ENDOR signal whereas the difference spectrum at I4 $\mathrm{MHz}$ has moved with the proton signals and is thus attributable to the effect of changing the proton relaxation times by the introduction of ${ }^{57} \mathrm{~F} \mathrm{e}^{\star}$. Referring again to the spectrum in Fig. Ioa, it is now possible to interpret the $17.5-\mathrm{MHz}$ line as due to a second iron site. Fig. Iob displays the ENDOR spectra obtained for ${ }^{57} \mathrm{Fe}$ and ${ }^{56} \mathrm{Fe}$ substituted proteins with the magnetic field set at $H_{\perp}$ - I6 Gauss. Notice that the difference spectrum has shifted to lower frequencies. Similarly, Fig. Ioc displays the ENDOR spectra when the magnetic field is set at $H_{\perp}$; again, the difference spectrum is shifted to still lower frequencies. This frequency dependence of the ${ }^{57} \mathrm{Fe}$ difference spectra can be followed continuously as the selected orientations move continuously from those for which the field is along the $z$-axis of the g-tensor to those for which the field is in the $x-y$ plane of the g-tensor principal axis system.
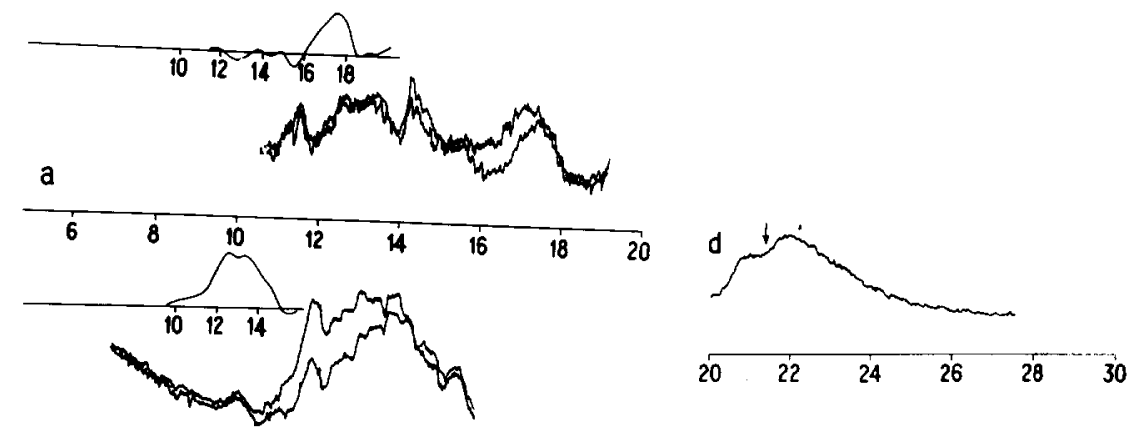

b

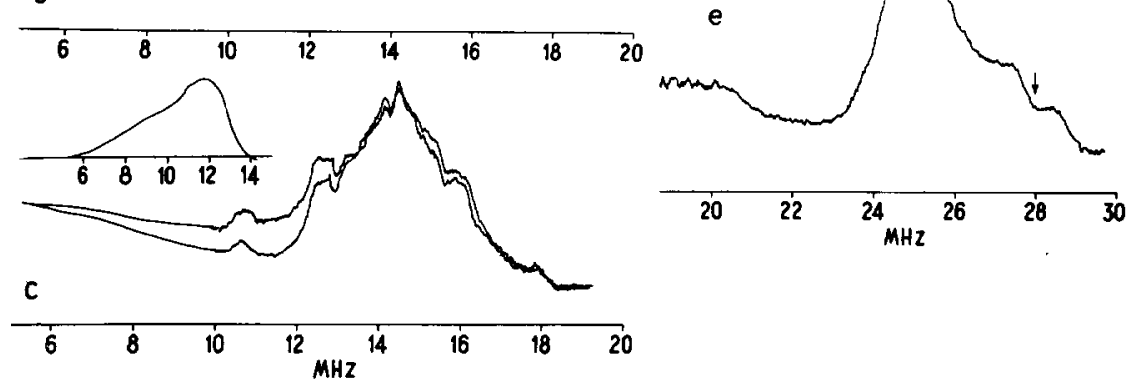

Fig. I2. The ENDOR spectra at $13^{\circ} \mathrm{K}$ and $v_{0}=925^{\circ} \mathrm{MHz}$ for reduced putidaredoxin in ${ }^{2} \mathrm{H}_{2} \mathrm{O}$ reconstituted with ${ }^{57} \mathrm{Fe}$ (upper trace) or ${ }^{56} \mathrm{Fe}$ (lower trace) with the magnetic field set at (a and $\mathrm{d}$ ) $H_{z}$, (b) $H_{\perp}-3$ I Grauss and (c and e) $H_{\perp}$.

Fig. I2 shows a similar set of ENDOR spectra obtained for ${ }^{56} \mathrm{Fe}$ and ${ }^{57} \mathrm{Fe}$ substituted putidaredoxin in the $6-30-\mathrm{MHz}$ region. Fig. I 2 a displays the spectra obtained when the magnetic field is set so as to select molecules whose $z$-axes are aligned along the applied field. Note the difference spectrum centered again around $\mathrm{I} 7.5 \mathrm{MHz}$ which can be attributed to ${ }^{57} \mathrm{Fe}$. Fig. I $2 \mathrm{~b}$ shows the spectra obtained when the magnetic field is set at $H_{\perp}-3 \mathrm{I}$ Gauss to select molecules of orientations intermediate between having the field along the $z$-axis and having it in the $x-y$ plane; here, the difference spectrum has shifted to lower frequencies. Similarly, Fig. I2c shows the

* See note added in proof on p. I32. 
ENDOR spectra obtained when the magnetic field is set at $H_{\perp}$ to select those molecules where the magnetic field is in the $x-y$ plane. The difference spectrum has shifted to still lower frequencies and is broad. Fig. I2d displays the extension of the ENDOR spectrum of the ${ }^{57} \mathrm{Fe}$ shown in Fig. I2a with the magnetic field chosen to select molecules with their $z$-axes along the field. The corresponding ${ }^{56} \mathrm{Fe}$ spectrum shows no absorption. The pair of lines centered at $2 \mathrm{I} .5 \mathrm{MHz}$ and separated by $0.9 \mathrm{MHz}$ is indicated by the arrow and may be attributed to ${ }^{57} \mathrm{Fe}$. Fig. Ize displays the extension of the ${ }^{57} \mathrm{Fe}$ spectrum shown in Fig. I2C with the magnetic field chosen so as to select molecules where the magnetic field is in the $x-y$ plane. Note the two pairs of lines centered at 25 and $28 \mathrm{MHz}$ indicated by the arrows; these will later be identified with the principal axis values of the hyperfine tensor in the $x-y$ plane. The absorption at 20 $\mathrm{MHz}$ is a proton line.

Fig. I3 displays the ENDOR spectra in the I0-30- $\mathrm{MHz}$ region obtained from the ${ }^{56} \mathrm{Fe}$ and ${ }^{57} \mathrm{Fe}$ substituted two-iron ferredoxin from parsley. Fig. I3a displays the spectra obtained when the magnetic field is set so as to select molecules whose $z$-axes

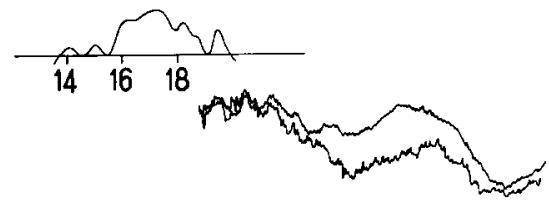

a
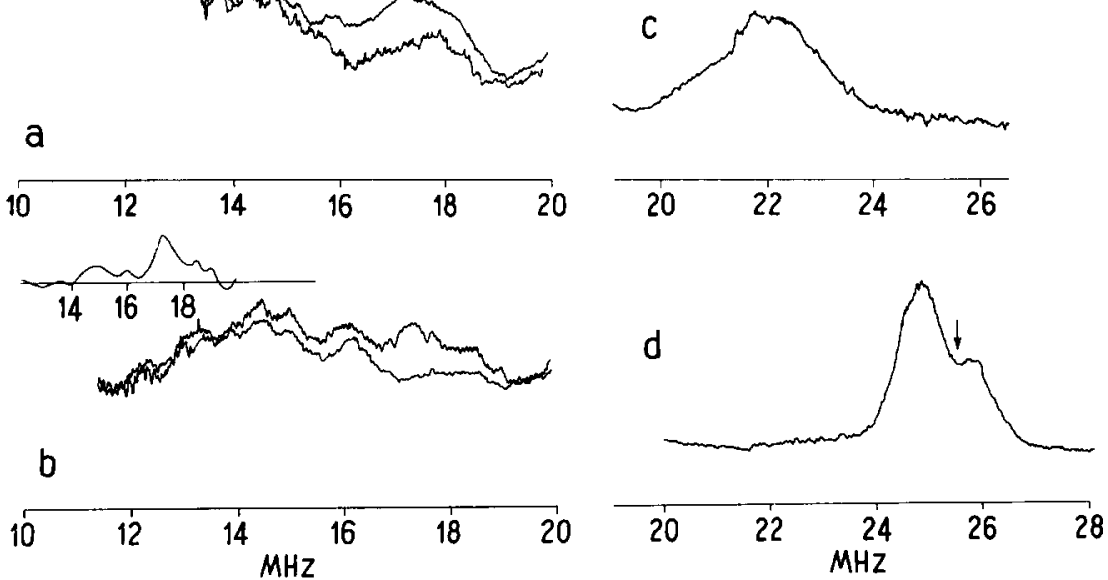

Fig. 13. The ENDOR spectra at $15^{\circ} \mathrm{K}$ and $v_{0}=9250 \mathrm{MHz}$ for reduced parsley ferredoxin in ${ }^{2} \mathrm{H}_{2} \mathrm{O}$, reconstituted with ${ }^{57} \mathrm{Fc}$ (upper trace or single trace) or ${ }^{56} \mathrm{Fe}$ (lower trace) and with the magnetic field set at (a and c) $H_{z}$, (b) $H_{z}+3$ I Gauss and (d) $H_{x}$. The difference spectra in (a) and (b) are plotted on a reduced scale in the upper left.

are aligned along the applied magnetic field. Note the difference spectrum again centered around $\mathrm{I} 7.3 \mathrm{MHz}$ which can be attributed to ${ }^{57} \mathrm{Fe}$. Fig. I3 $\mathrm{C}$ displays the second ${ }^{57} \mathrm{Fe}$ absorption obtained at the same magnetic field setting for these molecules and this is centered at $22 \mathrm{MHz}$. This latter absorption does not display the doublet character of the corresponding absorptions for the adrenal and putida proteins and this fact will be explained later. Fig. I3b shows the difference spectra obtained with the magnetic field set at $\mathrm{H}_{z}+3 \mathrm{I}$ Gauss - the difference spectrum decreases and stretches to lower frequency. When the magnetic field is set still closer to $H_{y}$ or $H_{x}$ no difference spectra can be detected in the $6-20-\mathrm{MHz}$ region. With the magnetic field set at $H_{x}$ to select molecules whose $x$-axis is along the applied field, the spectrum in Fig. I3d is 
obtained. Note the doublet once again. When the magnetic field is moved continuously from $H_{z}$ to $H_{x}$ the resulting spectra move continuously from that of Fig. I 3 b to that of Fig. I3d showing that these spectra belong to the same iron site. The difference spectrum in Fig. I3a is presumably from the other site.

Finally, the ENDOR spectra for the ${ }^{56} \mathrm{Fe}$ and ${ }^{57} \mathrm{Fe}$ substituted two-iron ferredoxin from spinach are displayed in Fig. I4. When the magnetic field is set at $H_{z}$ to select molecules where the applied magnetic field is along the $z$-axis the spectra in Figs. I $4 \mathrm{a}$ and $\mathrm{I} 4 \mathrm{C}$ are obtained. Again there is a difference spectrum centered at I7.7 $\mathrm{MHz}$ and a second absorption in the ${ }^{57} \mathrm{Fe}$ sample centered at $22 \mathrm{MHz}$. Just as for the parsley protein, there is no discernible doublet structure in the absorption at $22 \mathrm{MHz}$; however, when the magnetic field is changed to $H_{z}+3$ I Gauss the spectra displayed in Figs. $\mathrm{I}_{4} \mathrm{~b}$ and $\mathrm{I}_{4} \mathrm{~d}$ are obtained. Notice the doublet appearing at $2 \mathrm{I} .5 \mathrm{MHz}$ together with the absorption tailing to higher frequency and also the difference spectra in the $\mathrm{IO}-20-\mathrm{MHz}$ region which is getting weaker and possibly moving to lower frequencies. This same behavior is observed in the parsley protein. As the magnetic field is moved to higher values the difference spectrum disappears in the $6-20-\mathrm{MHz}$ region but the ${ }^{57} \mathrm{I}$ e spectrum in the $20-30-\mathrm{MHz}$ region persists. These spectra are displayed in Figs. I $4 \mathrm{e}$ and $\mathrm{I}_{4} \mathrm{f}$ for the field set at $\mathrm{H}_{z}+62$ Gauss and $H_{x}$, respectively. The doublet first seen in Fig. I4d at 2I.5 MHz persists and an additional doublet appears at $25 \mathrm{MHz}$ when the magnetic field is set at $H_{z}+62$ Gauss (Irig. I4e). When the magnetic field is inceased to $H_{x}$, only the doublet at $25.5 \mathrm{MHz}$ remains ( $\mathrm{Fig}$. I4f).
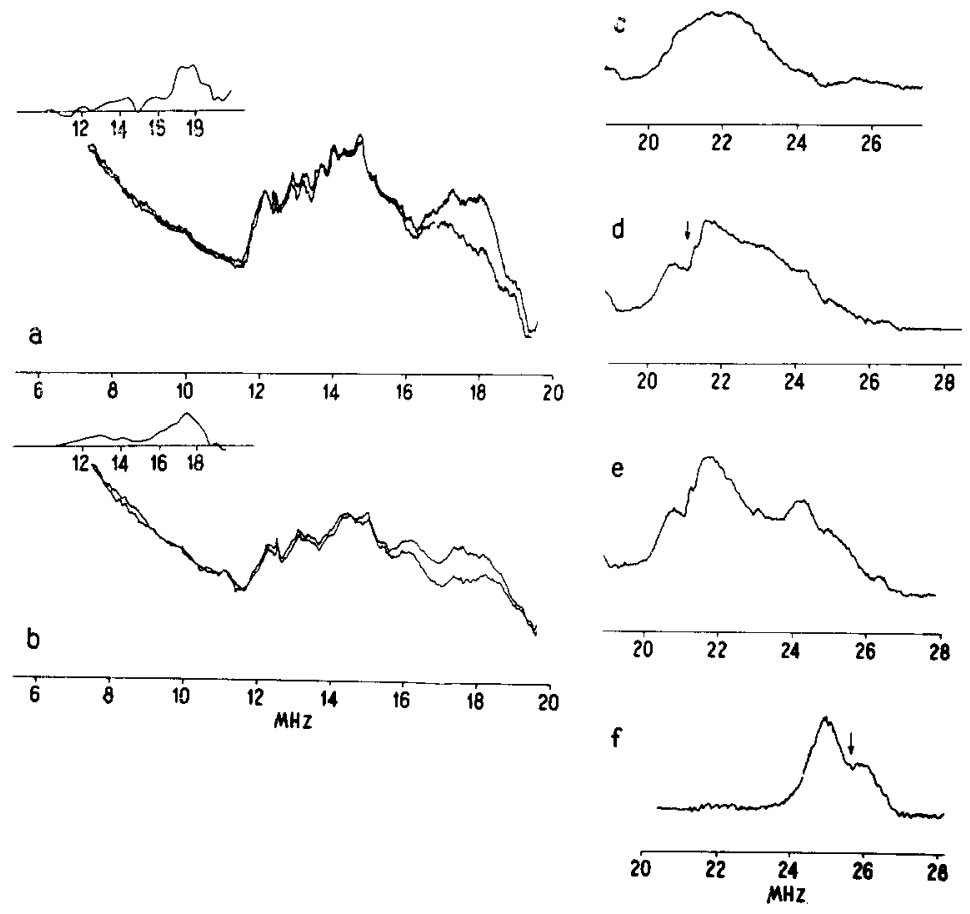

Fig. I4. The ENDOR spectra at $15^{\circ} \mathrm{K}$ and $\nu_{0}=9250.1 \mathrm{~Hz}$ for reduced spinach ferredoxin in ${ }^{2} \mathrm{H}_{2} \mathrm{O}$, reconstituted with ${ }^{57} \mathrm{Fe}$ (upper or single trace) or ${ }^{56} \mathrm{Fe}$ (lower trace) and with the magnetic field set at (a and c) $H_{z}$, (b and d) $H_{z}+3$ I Gauss, (e) $H_{z}+62$ Grauss and (f) $H_{x}$. The difference spectra in (a) and (b) are plotted on a reduced scale in the upper left. 
DISCUSSION

The first conclusion which can be reached from these ENDOR studies is that there are two clearly distinct iron sites in each of these proteins. This can be seen by examining the spectra taken with the magnetic field set to select molecules whose $z$-axes are aligned with the field (Figs. ga and roa for the ferredoxin from adrenal cortex, Figs. I2a and r2d for that from P. putida, Figs. I 3 a and $\mathbf{z} 3$ c for parsley ferredoxin and Figs. I4a and I4c for spinach ferredoxin). These spectra show ${ }^{57} \mathrm{Fe}$ absorption occurring at approximately $\mathrm{I} 7.5$ and $2 \mathrm{I} .5 \mathrm{MHz}$. These absorptions cannot be attributed to the same iron site since they are separated by more than twice the iron nuclear Zeeman frequency and the iron has no nuclear quadrupole moment which could be responsible for this splitting. The only interaction that can account for ENDOR signals in this frequency range is a hyperfine interaction with the net electronic spin. The EPR spectra (cf. Fig. 7) from adrenal cortex and P.putida ferredoxins demand two iron atoms with nearly equal $z$-components of their hyperfine tensors $\left(A_{z}\right)$ of approximately $40 \mathrm{MHz}$. These ENDOR spectra may be interpreted as coming from two iron atoms, one with $A_{z}=35 \mathrm{MHz}$ and the other with $A_{z}=43$ $\mathrm{MHz}$ (the corresponding ENDOR signals are centered about a frequency at $A_{z} / 2$ since the electronic spin is quantized by the electron Zeeman interaction to be along the applied field). Fig. 7 displays a computer fit to the previously published EPR spectrum assuming two inequivalent iron atoms having the $A$-tensors measured here (see Table I). Because the $z$-components of the $A$-tensor of the two iron atoms are within $20 \%$ of one another, a I:2: I triplet is observed at $H_{z}$ even though the two iron atoms are markedly non-equivalent as will be seen below. As the magnetic field used in the ENDOR instrument is changed to select molecules having different orientations with respect to that field, the resulting ENDOR spectra can be compared with computer simulated spectra and the corresponding $x$ - and $y$-components of the hyperfine tensors for each iron site can be deduced. The results are discussed below.

The second conclusion is that the iron atoms are interacting with an electronic system having a net spin of one-half $(S=1,2)$. That $S=1 / 2$ can be seen by noting that the ENDOR lines occur centered about $A / 2$ (cf. Figs. ga, I 2 d, I3d and I4f). Where the multiplicity can be resolved, the separation of the lines is just twice the nuclear Zeeman frequency for ${ }^{57} \mathrm{Fe}$ as shown in Fig. 4, thus providing additional confirmation that they are due to ${ }^{57} \mathrm{Fe}$.

The third conclusion concerns the relative orientations of the $A$ - and $g$-tensors for each iron site. This information is hidden in the data and to extract it requires a close comparison of the data with computer simulations. The first clue that the $A$ and $g$-tensor principal axes are not coincident can be seen in the comparison of Figs. $\mathrm{I} 4 \mathrm{C}$ and I4d for the spinach protein. When the magnetic field is set to select molecules whose g-tensor $z$-axes are along the field, no doublet is observed (Fig. I4c) indicating that there is a distribution of $A$-values, or rather that the $A$-value depends strongly on orientation. Since a distribution of orientations is always observed, due to finite EPR linewidths and " $g$-strains", there is a concomitantly large distribution of $A$-values. This last situation will be true if the $A$-tensor axes are rotated with respect to the g-tensor principal axes. Contrastingly, if the $A$-tensor ellipsoid has the same principal axes as the $g$-tensor ellipsoid, the variation of $A$ with orientation about one of the principal axes will be small hence a "single" $A$-value will be observed for all 
orientations resulting in a resolved doublet. Such doublets are always observed when the magnetic field is adjusted to select molecules whose $g$-tensor $x$-axes coincide with the applied field ( $c f$. Figs. I $3 \mathrm{~d}$ and I4f), hence it may be concluded that for the spinach and parsley proteins the $A$ - and $g$-tensor $x$-axes are nearly coincident (within I $5^{\circ}$, according to computer simulations).

Fig. I5 displays the computer simulations (dotted lines) which provided the best fit to the ENDOR spectra for iron site No. I in spinach ferredoxin. These are to be compared with the data of Figs. I4c, I4d, I4e and I4f (solid lines in Figs. I 5a, I 5 b, $\mathrm{I}_{5} \mathrm{c}$, and $\mathrm{I}_{5} \mathrm{f}$, respectively). The reader's attention is called to Figs. I5 a and $\mathrm{I}_{5} \mathrm{~b}$ where the doublet does not appear at $H_{z}$ but does appear at $H_{z}+3 \mathrm{I}$ Gauss. This doublet centered at $2 \mathrm{I} .5 \mathrm{MHz}$ indicates the principal axis value $A_{z}{ }^{\prime}$ to be $42 \mathrm{MHz}$ with the $z^{\prime}$-axis tilted from the $z$-axis of the $g$-tensor by approximately $30^{\circ}$ - this $30^{\circ}$ rotation is about the coincident $x$-axes of the $A$ - and $g$-tensors as indicated above. A similarly assumed rotation of the $A$-tensor with respect to the $g$-tensor gives a good fit for parsley. In this computer simulation it was necessary to adjust the values for $A_{x}$, $A_{y}{ }^{\prime}$ and $A_{z}{ }^{\prime}$ as well as the Euler angles describing the $A$ - and $g$-tensor relative orientation to obtain a best fit. It must be noted that this agreement between theory and experiment is amazingly good everywhere but at $H_{z}+$ I20 Gauss (Fig. I5d). Close to this field value the computer program develops a well resolved doublet centered at $25 \mathrm{MHz}$ which is not seen in the corresponding data. We have no explanation of this
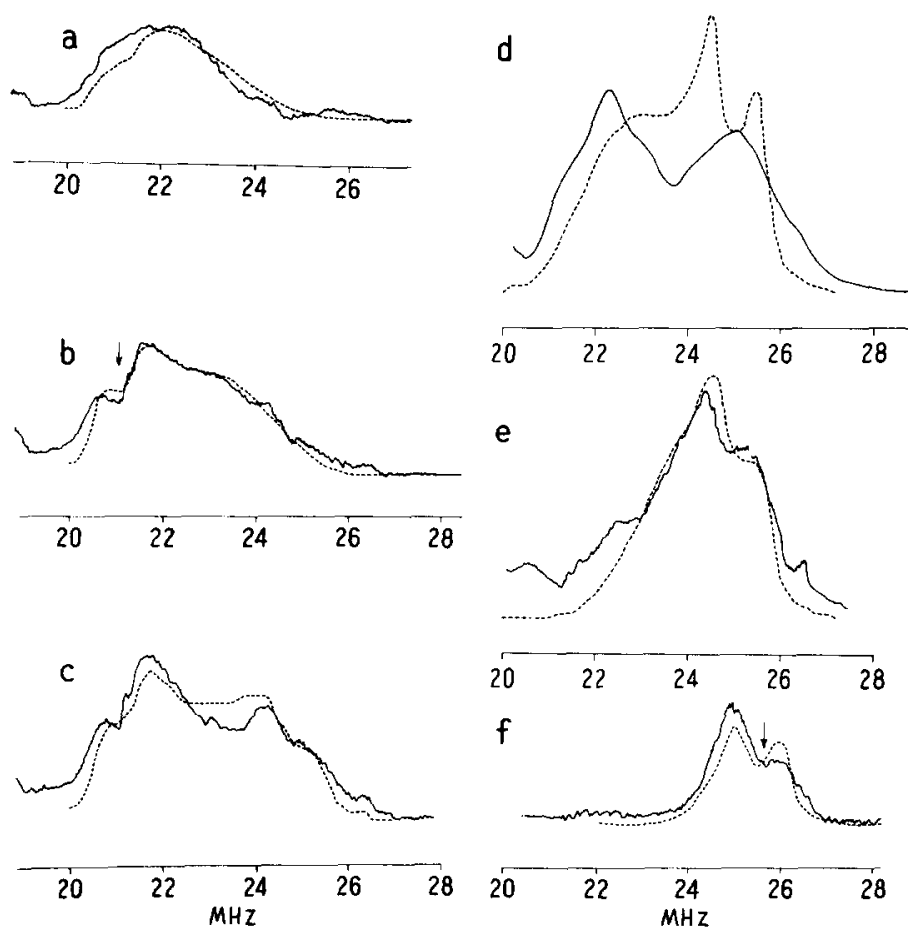

Fig. I5. A comparison of the experimental (_- - ) and computer simulated(---) ENDOR spectra for reduced ${ }^{57} \mathrm{Fe}$ reconstituted spinach ferredoxin in the $20--28-\mathrm{MHz}$ region (site No. I) with the magnetic field set at (a) $H_{z}$, (b) $H_{z}+3$ I Gauss, (c) $H_{z}+62$ Gauss, (d) $H_{z}+$ I 20 Gauss, (e) $H_{x}-$ Ioo Gauss and (f) $H_{x}$. 
discrepancy which we can prove conclusively; nevertheless, it is our feeling that the rotation of the $A$-tensor axes relative to the $g$-tensor axes presented here is basically correct. The resulting parameters, together with the extremum error flags are tabulated in Table I, where the $A$-values tabulated for iron site No. I are the principal axes values. For the spinach and parsley proteins these $A$-tensor principal axes for site No. I are rotated with respect to the $g$-tensor principal axes by a rotation of $30^{\circ}$ about the coincident $g$ - and $A$-tensor $x$-axes. For the adrenal cortex and $P$. putida proteins the $A$-tensor and $g$-tensor $z$-axes for site No. I are coincident or nearly so and because this $z$-axis is the $g$-tensor axis of symmetry no rotation about this axis has any physical significance - thus the $g$ - and $A$-tensors for the seproteins may be assumed coincident. Notice, however, that there are two principal axis $A$-values in the $x-y$ plane of the $g$-tensor, labeled $A_{\perp}$ and $A_{\perp}{ }^{\prime}$ indicating that there is not an axis of symmetry for the iron site No. I in these proteins despite the fact the $g$-tensor is axially symmetric. The errors in these two values are indicated by \pm and $\mp$, respectively, in Table $I$, which is meant to imply that if $A_{\perp}$ is error by a positive $(+)$ increment then $A_{\perp}^{\prime}$ must be corrected by a negative $(-)$ increment or vice versa. The poor signal-to-noise for the ENDOR signals of iron site No. 2 does not permit the relative orientations of the $g$ - and $A$-tensors for this site to be determined, hence the $A$-values tabulated are "effective" $A$-values for this site assuming a set of axes such that the $z$-axis is along the $g$-tensor $z$-axis and the other axes are somewhere in the $x-y$ plane of the g-tensor principal axes.

\section{CONCLUSIONS}

From these data it is evident that each protein contains two highly non-equivalent iron atoms, each having a sizeable hyperfine interaction with the net electronic angular momentum $(S=1 / 2)$ in the reduced state. Detailed information about the hyperfine tensors results from these ENDOR studies.

By employing these measured $A$-values in the computer simulated Mössbauer spectra $^{25}$ in Paper II, it will be shown that iron site No. I corresponds to a high spin $(S=5 / 2)$ ferric (FeIII)) atom and iron site No. 2 to a high $\operatorname{spin}(S=2)$ ferrous (FeII $))$ atom, antiferromagnetically coupled to form a net spin one-half $(S=1 / 2)$ system. For such a system it is possible to express the effective $A$-values for the complex in terms of the actual $A$-values for the individual atoms and hence to ascertain the degree of covalency for each iron atom and to demonstrate the consistency of these measured $A$-values with such an interpretation. This will be done in Paper II. Suffice it to say that these measured effective $A$-values are essential to obtaining a unique fit to the Mössbauer spectra and hence this paper precedes the Mössbauer work to follow. The analysis of the Mössbauer data permitted by these measured $A$-values will allow us to conclude the location of the reducing electron and its role in the determination of the molecular paramagnetism.

The presence of the proton ENDOR signals (shown in Fig. 8) allows for the possibility that these protons can be located with respect to the complex. The interpretation of these signals is proceeding along with efforts to detect other ligand $A$-tensors and the results will be reported at a later date. 


\section{ACKNOWLEDGEMENTS}

We wish to acknowledge the considerable moral and technical support given by Dr. James S. Hyde of Varian Associates, without which we might still be troubleshooting our apparatus. We are indebted also to Dr. Irving Salmeen for writing the first ENDOR computer program employed by us.

The research herein described was supported in the large by National Institutes of Health Grants (GM-I2I76) and (RR-O04I7-OI). Auxiliary support was obtained under National Institutes of Health Grant (AM-00562) to I.C.G., a Public Health Service Research Career Award to H.B. (5-Ko6-GM-I8,442) and Public Health Service Research Career Development Awards to G.P. (I-K3-GM-2I,213), to J.C.M.T. (I-K4-AM-42386) and W.H. O-J. (I-K3-GM-I0,236) and for R.A. and J.F. the Public Health Service National Institutes of Health, Biophysics Training Grant GM-I355.

\section{NOTE ADDED IN PROOF: (Received September 7 th, I97I)}

We have since discovered that the ${ }^{56} \mathrm{Fe}$ sample was reconstituted in ${ }^{2} \mathrm{H}_{2} \mathrm{O}$ whereas the ${ }^{57} \mathrm{Fe}$ sample was reconstituted in ${ }^{1} \mathrm{H}_{2} \mathrm{O}$ and later placed in ${ }^{2} \mathrm{H}_{2} \mathrm{O}$. This could account for the observed differences in this proton spectra.

\section{RLFERENCES}

I D. O. Hall and M. C. W. Lvans, Nature, 223 (I969) I 342.

2 G. Palmer and H. Brintzinger, in T. King and M. Klingenberg, A Treatise on Electron Transport, Vol. I, Marcel Dekker, New York, I97 I, (in the press).

3 J. C. M. Tsibris and R. W. Woody, Structural Studies of Iron-Sulfur Proteins, Coord. Chin. Rev., $5(1970) 417-458$.

4 A. M. Benson and K. T. Yasunobu, J. Biol. Chem., 244 (1966) 955.

5 S. Keresztes-Nagy, F. Perini and E. Margoliash, J. Biol. Chem., 244 (1969) 98 i.

6 H. Matsubara, R. M. Sasakl and R. K. Chatn, J. Biol. Chem., 243 (I968) I 725.

7 K. Sugeno and H. Matsubara, Biochem. Biophys. Res. Commun., 32 (1968) 95 I.

8 T. H. Moss, D. Petering and G. Palmer, J. Biol. Chem., 244 (1969) 2275.

9 T. Kimura, A. Tasaki and H. Watari, J. Biol. Chem., 245 (I970) 4450.

io C. Moleski, T. H. Moss, W. H. Orme-Johnson and J. C. M. Tsibris, Biochim. Biophys. Acta, $214(1970) 548$.

i H. Brintzinger, G. Palmer and R. H. Sands, Proc. Natl. Acad. Sci., U.S., 55 (ig66) 397.

I2 Y. I. Shethna, P. W. Wilson, R. E. Hansen and H. Beinert, Biochim. Biophys, Acta, I 3 (1966) 225.

i 3 H. Beinert and W. H. Orme-Johnson, Ann. N.Y.Acad. Sci., 158 (rg6g) 336.

i 4 W. H. Orme-Johnson, R. E. Hansen and H. Beinert, Fed. Proc, 27 (1986) 298.

i 5 W. H. Orme-Johnson, R. E. Hansen, H. Beinert, J. C. M. Tsibris, R. C. Bartholomaus and I. C. Gunsales, Proc. Natl. Acad. Sci. L.S., 60 (1968) 368.

i6 J. C. M. Tsibris, R. L. Tsai, I. C. Gunsalus, W. H. Orme-Johnson, R. E. Hansen ani: H. Beinert, Proc. Natl. Acad. Sci. U.S., 59 (1968) 959.

I7 T. C. Hollocher, F. Solomon and T. E. Ragland, J. Biol.Chem, 24 I (Ig66) 3452.

is D. V. DerVartanian, W. H. Orme-Johnson, R. E. Hansen, H. Beinert, R. L. Tsai, J. C. M. Tsibris, R. C. Bartholomaus and J. C. Gunsalus, Biochem. Biophys. Res. Commun., 26 (1967) 560.

ig W. E. Blumberg and J. Peisach, in A. San Pietro and L. P. Vernon, Non-Heme Iron Proteins, Role in Enevgy Conversion, Antioch Press, Yellow Springs, Ohio, I965, p. Ior.

20 J. D. W. Van Voorst and P. Hemmerich, J. Chem. Phys., 45 (I966) 3914.

21 H. Beinert, D. V. DerVartanian, P. Hemmerich, C. Veeger and J. D. W. Van Voorst, Biochim. Biophys. Acta, 96 (I965) 530.

22 H. Brintzinger, G. Palmel and R. Sands, Proc. Natl. Acad. Sci. U.S., 55 (1966, 397.

23 J. F. Gibson, D. O. Hall, J. H. M. Thornley and I'. R. Whatley, Proc. Natl. Acad. Sci. U.S., $50($ I 966$) 987$. 
24 J. H. M. Thornley, J. F. Gibson, F. R. Whatley and D. O. Hall, Biochem. Biophys. Res. Commun., 24 (I966) 877.

25 W. R. Dunham, A. Bearden, I. Salmeen, G, Palmer, R. H. Sands, W. H. Orme-Johnson And H. Beinert, Biochim. Biophys. Acta, 253 (I97I) I 34.

26 G. Palmer, W. R. Dunham, J. A. Fee, R. H. Sands, 'T. Iizuka and T. Yonetani, Biochim. Biophys. Acta, 245 (197I) $20 \mathrm{I}$.

27 W. Eaton, G. Palmer, J. A. Fee, T. Kimura and W. Lovenberg, Proc. Natl. Acad. Sci. U.S., submitted.

28 W. R. Dunham, G. Palmer, R. H. Sands and A. Benrden, Biochim. Biophys. Acta, in the press.

29 D. Petering and G. Palmer, Arch. Biochem. Biophys., i 4 I (I970) $45^{6}$.

30 J. A. Fee and G. Palmer, Biochim. Biophys. Acta, 245 (r97 I) I 75.

3 I J. C. M. Tsibris, R. L. Tsai, I. C. Gunsalus, W. H. Orme-Johnson, R. E. Hanson and H. Betnert, Proc. Natl. Acad. Sci. U.S., 59 ( I968) 959.

32 W. H. Orme-Johnson And H. Beinert, I. Biol. Chem., 244 (I969) 6r 43.

33 H. Beinert and W. H. Orme-Johnson, Ann. N.Y. Acad. Sci., I 58 (I969) 336.

34 G. Palmer, Biochem. Biophys. Res. Commun. 27 (1967) 3 I 5.

35 H. Beinert, in W. Lovenberg, Iron-Sulfur Proteins, Academic Press New York, in the press.

36 S. G. Mayhew, D. Petering, G. Palmer and G. P. Foust, J. Biol. Chem., 244 (1969) 2830.

37 G. Feher, Phys. Rev., II 4 (I959) I2I9.

38 J. S. Hyde and A. H. Maki, J. Chem. Phys., $40($ J964) 3 II 7.

39 J. S. Hyde, J. Chem. Phys., 43 (I965) I 806.

4 G. H. RIST AND J. S. HYDE, J. Chem. Phys., 52 (1970) 4633 and earlier publications referenced in this paper.

4 I A. Abragam nnd B. Bleaney, Electron Paramagnetic Resonance of Transition Ions, Clarendon Press, Oxford, England, 1970, Chapter 4.

42 IF. K. Kneubühl, J. Chem. Phys., 33 (1960) Io74.

43 IF. K. Kneubühl and B. Natterer, Helv. Phys. Acta, 34 (I96i) 7 Io.

44 R. Neuman and D. Kivelson, J. Chem. Phys., 35 (1961) I 49.

45 R. Neuman and D. Krvelson, J. Chem. Phys., 35 (ig6r) i 56.

46 H. R. Gersmann and J. D. Swalen, J. Chem. Phys., 36 (Ig62) 322 I.

47 M. M. Malley, J. Mol. Spectrose., 17 (1965) 2 Io.

$4^{8}$ T. S. Johnson and H. G. Hecht, J. Mol. Spectrosc. , i 7 (1965) 98.

49 J. S. Hyde, in A. Ehrenberg, B. G. Malmstrom, And T. Vanngard, Magnetic Resonance in Biological Systems, Pergamon Press, London, I967, p. 63, 82.

50 R. H. SANDS AND R. ANDERSON, in preparation.

Biochim. Biophys. Acta, 253 (1971) I10-133 